Esther Klann, Ronny Ramlau

\title{
Regularization properties of Mumford-Shah- type functionals with perimeter and norm constraints for linear ill-posed problems
}

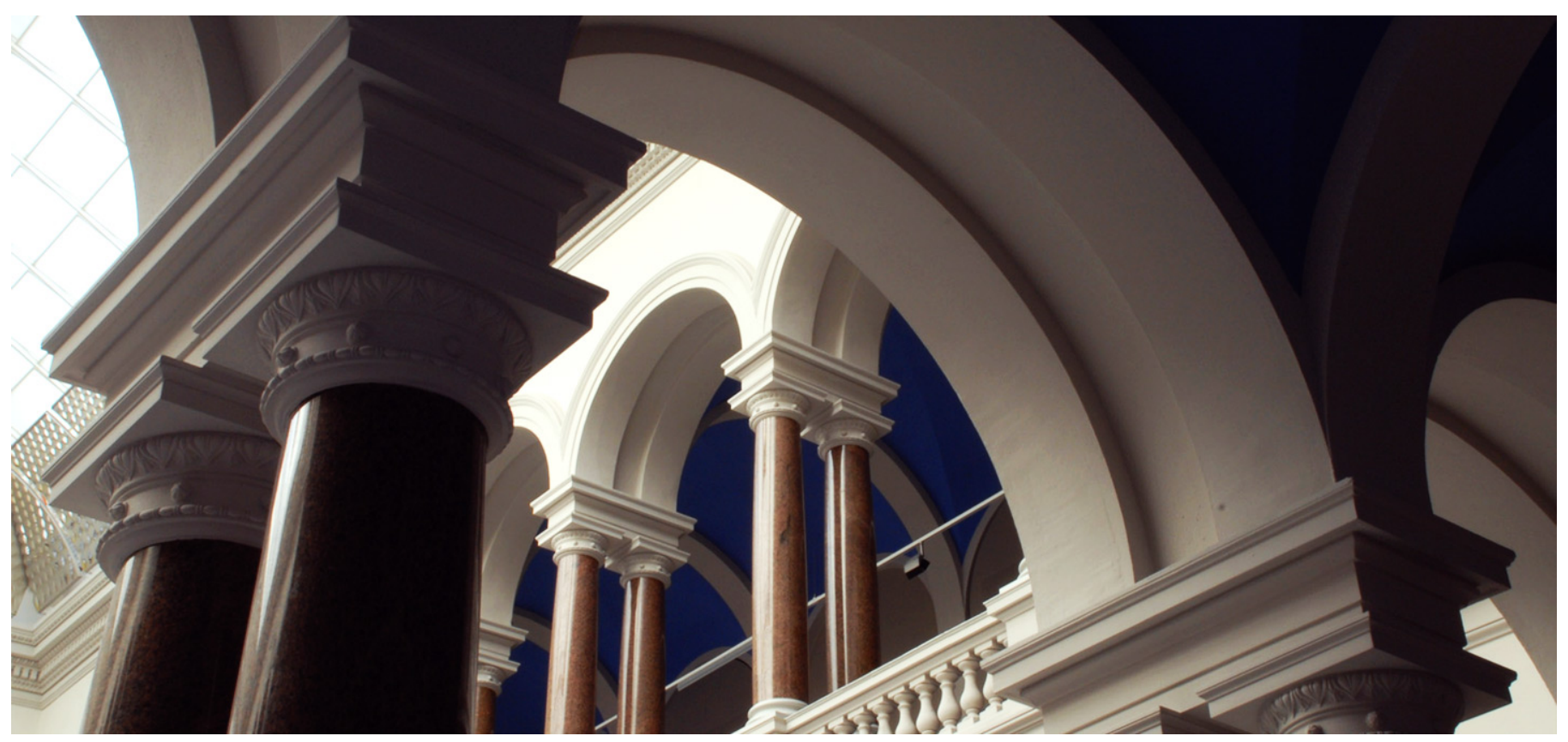

First Published in SIAM journal on imaging sciences in 6 (2013), 1, pp. 413-436, published by the Society for Industrial and Applied Mathematics (SIAM). DOI: 10.1137/110858422 


\title{
REGULARIZATION PROPERTIES OF MUMFORD-SHAH TYPE FUNCTIONALS WITH PERIMETER AND NORM CONSTRAINTS FOR LINEAR ILL-POSED PROBLEMS
}

\author{
ESTHER KLANN* AND RONNY RAMLAU
}

\begin{abstract}
In this paper we consider the simultaneous reconstruction and segmentation of a function $f$ from measurements $g=K f$, where $K$ is a linear operator. Assuming that the inversion of $K$ is ill-posed, regularization methods have to be used for the inversion process in case of inexact data. We propose to use a Mumford-Shah type functional for the stabilization of the inversion. Restricting our analysis to the recovery of piecewise constant functions, we investigate the existence of minimizers, their stability and the regularization properties of our approach. Finally we present a numerical example from Single Photon Emission Computed Tomography (SPECT).
\end{abstract}

Key words. regularization, ill-posed problems, Mumford-Shah, SPECT

AMS subject classifications. 35R25, 65F $22,44 \mathrm{~A} 12,65 \mathrm{~K} 10,92 \mathrm{C} 50,94 \mathrm{~A} 08$

1. Introduction. In this article, we study the regularization properties of a Mumford-Shah type functional for ill-posed operator equations

$$
K f=g .
$$

We assume that $K$ is a linear operator between Hilbert spaces $X$ and $Y$ (usually $L_{2}$ ) and does not have a bounded inverse. Furthermore, we assume that instead of the exact data $g$, only a noisy version $g^{\delta}$ with known noise level $\delta$ is available. In such a situation, regularization methods have to be applied in order to achieve a stable reconstruction. In standard methods one aims for the reconstruction of $f$ in terms of function values only. In contrast to this we reconstruct the singularity set of $f$, i.e., the set of points where the function $f$ is discontinuous, as well as averaged function values of the function away from the singularities. To be more concrete, we assume that the solution $f$ is defined on some domain $D \subset \mathbb{R}^{2}$ and can be represented as a piecewise constant function, i.e.,

$$
f=\sum_{i \in I} \mathrm{f}_{i} \chi_{\Omega_{i}}
$$

where $I$ is a finite index set, $\mathrm{f}_{i} \in \mathbb{R}$ and $\bigcup_{i \in I} \Omega_{i}=D$ with pairwise disjoint sets.

For the presented approach we define a solution to consist of a sequence $\boldsymbol{\Omega}$ of sets $\Omega_{i} \subset \mathbb{R}^{2}$ with $\bigcup_{i \in I} \Omega_{i}=D$ and a vector $\mathbf{f}$ of coefficients $\mathrm{f}_{i} \in \mathbb{R}$. In doing so, we achieve a segmentation of $f$ defined by $\boldsymbol{\Omega}$ and a reconstruction of $f$ as in (1.2). In order to find an approximation to our problem we consider the Mumford-Shah type functional

$$
J_{\beta, \gamma}(\boldsymbol{\Omega}, \mathbf{f})=\left\|K f-g^{\delta}\right\|^{2}+\beta\|\mathbf{f}\|^{2}+\gamma \sum_{i \in I}\left|\partial \Omega_{i}\right|
$$

where $\|\mathbf{f}\|^{2}$ denotes a norm penalty on the coefficient vector $\mathbf{f}$, and $\left|\partial \Omega_{i}\right|$ denotes the length of the boundary of the set $\Omega_{i}$. A solution to the problem of simultaneous reconstruction and segmentation is defined as minimizing argument of this functional, see also (2.8) and (2.9).

\footnotetext{
*The work of this author was partially supported by FWF-grant T529-N18
} 
The paper is mainly concerned with an analysis of the above functional. In particular, we show existence and stability of its minimizers, see Section 5 . We also propose a rule for choosing the regularization parameters $\beta$ and $\gamma$ that ensures convergence of the sequence of minimizers $\left(\boldsymbol{\Omega}_{\beta, \gamma}^{\delta}, \mathbf{f}_{\beta, \gamma}^{\delta}\right)$ to the exact solution $\left(\boldsymbol{\Omega}^{\dagger}, \mathbf{f}^{\dagger}\right)$ as $\delta \rightarrow 0$.

Our approach can be understood as a generalization to the Mumford-Shah type functional that was considered in [32]. The significant difference is our choice of regularization terms. In [32], a reduced Mumford-Shah type functional with only the second penalty term, i.e., the perimeter regularization, was used. This causes problems in the analysis of the method. In particular, convergence could only be shown under additional assumptions on the solutions.

Inverse Problems, where the variable to be reconstructed is a shape, have been considered for a long time. We refer to [32] for an overview on the related research. The Mumford-Shah functional as objective functional allows to reconstruct functional and geometrical parameters simultaneously in contrast to pure shape identification problems. It was originally used with the identity operator in place of the operator $K$, designed to identify the singularities in, e.g., an image and to provide at the same time a smooth (denoised) approximation to the image away from the singularities $[30,5,7,17,24]$. A non-trivial operator $K$ in the Mumford-Shah functional was - to our knowledge - first treated in [33] using an Ambrosio-Tortorelli type approximation of the Mumford-Shah functional. In [12] the Mumford-Shah idea was used for geometric image registration and in [31] and [19] the operator under consideration was the Radon transform and the attenuated Radon transform of computerized tomography. In these three papers, the Mumford-Shah model was interpreted as a shape identification problem over a restricted class of admissible shapes. In [2], the authors combine semi-blind deconvolution (parametric blur kernel) and Mumford-Shah segmentation.

In our paper, we are in particular interested in regularization properties of the considered Mumford Shah functional, i.e., in parameter choice rules that ensure convergence of the approximate solutions. Burger [4] used Morozov's discrepancy principle in order to identify an indicator function from noisy measurements of a linear system and proved that the approximations obtained by a level set approach converge to the original set. Ben Ameur et.al. [3] considered a Mumford-Shah functional for a linear elasticity problem. Equipped with an a priori rule for the regularization parameter they proved convergence of the reconstructed shapes in the Haussdorf metric. Leitao and Scherzer proposed a variational approach towards regularization by level-set methods, where the authors also penalized the level-set function and showed convergence in a weak sense [22,13]. DeCezaro et.al.[10] analyzed a multiple level-set method regarding existence, stability and convergence of minimizer of a corresponding Tikhonov functional. In most geometrical inverse problems the structure of the optimal shape is not known a-priori, and methods that allow a topology change have to be used. A well established approach for this is the level set method [23, 27, 28, 29, 35], where the shape of the object is encoded by means of a level-set function.

Analytic and geometric properties of minimizers of the Mumford-Shah functional have been studied extensively in the last years (see, e.g., the comprehensive exposition $[9]$ and the references therein). However, analytical investigations from the perspective of regularization theory of ill-posed problems are rather rare.

We finish our review with a few remarks on BV regularization as introduced in [34]. As this methods prefers the reconstruction of piecewise constant functions, it seems to fit to our problem. A BV-functional with a linear operator has been studied, 
e.g., in [16]. However, the reconstruction is still a functional one, i.e., a segmentation is not simultaneously done with the inversion procedure and has to be done afterwards. For a numerical comparison of the two methods we refer to [32].

The paper is organized as follows. In Section 2 we define the Mumford-Shah type functional and introduce basic notation concerning perimeter, partitions and piecewise constant functions. In Section 3 we define convergence of piecewise constant functions and present a result on the compactness of partitions with finite perimeter. In Section 4 we compute optimal coefficients of the Mumford-Shah type functional for fixed geometry as well as their behavior if a convergent sequence of partitions is given. In Section 5 we present the main results on regularization of linear ill-posed problems by the described Mumford-Shah type method. In Section 6 we apply the proposed method to a problem from medical imaging (SPECT with known density function) and demonstrate numerically that the proposed parameter choice rule yields a convergent scheme.

2. The Mumford-Shah type functional. In order to define the functional for which we are going to prove existence, stability and convergence of minimizers we start with a specification of the solutions we want to reconstruct.

We aim for the reconstruction of a piecewise constant function $f: D \rightarrow \mathbb{R}$, compare equation (1.2). The geometrical part of the reconstruction, i.e., the segmentation, is described by a collection of finite perimeter or Caccioppoli sets which form a partition of the domain of definition of the function.

Definition 2.1. Let $D$ be a domain in $\mathbb{R}^{d}$ and $\Omega$ a measurable subset of $D$. The perimeter of $\Omega$ in $D$ is defined as

$$
\begin{aligned}
p_{D}(\Omega):=\sup \{ & \int_{\Omega} \operatorname{div}(v(x)) d x ; v: D \rightarrow \mathbb{R}^{d}, \\
& \left.v \text { smooth with } \operatorname{supp}(v) \subset D \text { and }\|v(x)\|_{\infty} \leq 1 \forall x \in D\right\} .
\end{aligned}
$$

A measurable set $\Omega$ with $p_{D}(\Omega)<\infty$ is called a set of finite perimeter or a Caccioppoli set.

Later on, we also need the measure theoretic boundary and some relation between the perimeter and the Hausdorff measure of a set.

Definition 2.2 (Measure-theoretic boundary). Let $D \subset \mathbb{R}^{d}$ be a Lebesgue measurable set, i.e., $\mu(D)<\infty$. Then, the measure-theoretic boundary of $D$ is defined as

$\partial_{M} D:=\left\{x: \lim \sup _{r \rightarrow 0} \frac{\mu(D \cap B(x, r))}{\mu(B(x, r))}>0\right\} \cap\left\{x: \limsup _{r \rightarrow 0} \frac{\mu\left(\left(\mathbb{R}^{d} \backslash D\right) \cap B(x, r)\right)}{\mu(B(x, r))}>0\right\}$.

Definition 2.3 (Hausdorff-measure). For each $\gamma \geq 0, \varepsilon>0, \alpha(\gamma)>0$ and $\Omega \subset \mathbb{R}^{d}$, let

$$
H_{\varepsilon}^{\gamma}(\Omega):=\inf \left\{\sum_{i=1}^{\infty} \alpha(\gamma) 2^{-\gamma} \operatorname{diam}\left(A_{i}\right)^{\gamma}: \Omega \subset \bigcup_{i=1}^{\infty} A_{i}, \operatorname{diam}\left(A_{i}\right)<\varepsilon\right\} .
$$

As $H_{\varepsilon}^{\gamma}$ is decreasing in $\varepsilon$ the limit for $\varepsilon \rightarrow 0$ exists and the $\gamma$-dimensional Hausdorff measure of $\Omega$ is defined by

$$
H^{\gamma}(\Omega):=\lim _{\varepsilon \rightarrow 0} H_{\varepsilon}^{\gamma}(\Omega)
$$


If $\gamma=s \in \mathbb{N}, \alpha(\gamma)$ denotes the volume of the unit ball in $\mathbb{R}^{s}$.

The perimeter and the Hausdorff measure of a set are related as follows, e.g., [1].

Proposition 2.4 (Perimeter and Hausdorff measure).

1. For an open set $\Omega \subset D$ with a $\mathcal{C}^{1}$-smooth boundary it is

$$
p_{D}(\Omega)=\mathcal{H}^{n-1}(\partial \Omega \backslash \partial D) .
$$

2. For a Caccioppoli set $\Omega \subset D$ it is

$$
p_{D}(\Omega)=\mathcal{H}^{n-1}\left(\partial_{M} \Omega\right) .
$$

Definition 2.5 (Partition). Let $D \subset \mathbb{R}^{d}$ be a domain and $I \subset \mathbb{N}$ an index set. A collection of Caccioppoli sets $\Omega_{i} \subset D$ with $\mu\left(\Omega_{i}\right)>0$ for $i \in I$ forms a partition of $D$ if

$$
\chi_{D}=\sum_{i \in I} \chi_{\Omega_{i}}
$$

in $L_{1}(D)$. As the standard notation for a partition we use $\boldsymbol{\Omega}=\left(\Omega_{i}\right)_{i \in I}$.

From now on we restrict our reconstructions to partitions consisting of finitely many sets.

Definition 2.6. Let $D \subset \mathbb{R}^{d}$ be a domain and $M \in \mathbb{N}$. The set of partitions of $D$ of at most $M$ components is defined as

$$
\mathcal{G}_{M}(D):=\left\{\boldsymbol{\Omega}: \boldsymbol{\Omega}=\left(\Omega_{i}\right)_{i \in I} \text { is a partition of } D \text { with } \# I \leq M\right\} .
$$

We denote the number of sets in a partition $\boldsymbol{\Omega}$ with $m_{\boldsymbol{\Omega}}$, i.e., it is $\# I=m_{\boldsymbol{\Omega}}$.

A piecewise constant function $f: D \rightarrow \mathbb{R}$ is determined by a partition $\boldsymbol{\Omega} \in \mathcal{G}_{M}(D)$ and a coefficient vector $\mathbf{f}=\left(\mathrm{f}_{1}, \ldots, \mathrm{f}_{m_{\Omega}}\right) \in \mathbb{R}^{m_{\Omega}}$ via the mapping

$$
\begin{aligned}
F: \mathcal{G}_{M}(D) \times \mathbb{R}^{m_{\boldsymbol{\Omega}}} & \rightarrow L_{2}(D) \\
F(\boldsymbol{\Omega}, \mathbf{f}) & :=\sum_{i=1}^{m_{\boldsymbol{\Omega}}} \mathrm{f}_{i} \chi_{\Omega_{i}} .
\end{aligned}
$$

Definition 2.7. The (total) perimeter of a partition $\boldsymbol{\Omega} \in \mathcal{G}_{M}(D)$ is defined as

$$
|\partial \boldsymbol{\Omega}|:=\sum_{i=1}^{m_{\Omega}} p_{D}\left(\Omega_{i}\right)
$$

where $p_{D}(A)$ denotes the perimeter of $A \subset D$.

We formulate the Mumford-Shah type functional with perimeter and norm constraints for the reconstruction of a piecewise constant function as

$$
J_{\beta, \gamma}(\boldsymbol{\Omega}, \mathbf{f})=\left\|K F(\boldsymbol{\Omega}, \mathbf{f})-g^{\delta}\right\|_{L_{2}}^{2}+\beta\|\mathbf{f}\|_{\ell_{2}}^{2}+\gamma|\partial \boldsymbol{\Omega}|,
$$

where $\beta$ and $\gamma$ are non-negative regularization parameters. As a regularized solution of the problem of simultaneous reconstruction and segmentation we define

$$
\left(\boldsymbol{\Omega}_{\beta, \gamma}, \mathbf{f}_{\beta, \gamma}\right):=\underset{\mathcal{G}_{M}(D) \times \mathbb{R}^{m} \boldsymbol{\Omega}}{\arg \min } J_{\beta, \gamma}(\boldsymbol{\Omega}, \mathbf{f}) .
$$


3. Compactness of partitions. For two measurable sets $\Omega_{1}, \Omega_{2} \subset D$ we define the distance

$$
d_{L_{1}}\left(\Omega_{1}, \Omega_{2}\right)=\left\|\chi_{\Omega_{1}}-\chi_{\Omega_{2}}\right\|_{L_{1}(D)} .
$$

The set of equivalence classes of Lebesgue measurable subsets of $D$ which differ only by a set of measure zero, together with the distance function $d_{L_{1}}$ is a complete metric space (see, [11, Thm.2.1; p.93]). In the following we are not going to distinguish between the equivalence classes and their individual representatives. Moreover, we will write

$$
\Omega_{n} \stackrel{d_{L_{1}}}{\longrightarrow} \Omega \Longleftrightarrow d_{L_{1}}\left(\Omega_{n}, \Omega\right) \rightarrow 0
$$

for the convergence of sets in the sense of characteristic functions.

Definition 3.1. A sequence $\left(\boldsymbol{\Omega}^{n}\right)$ of partitions $\boldsymbol{\Omega}^{n}=\left(\Omega_{i}^{n}\right)_{i=1}^{m_{n}}$ converges to a partition $\boldsymbol{\Omega}=\left(\Omega_{i}\right)_{i=1}^{m}$, in short $\boldsymbol{\Omega}^{n} \rightarrow \boldsymbol{\Omega}$, if

1. $\exists N \in \mathbb{N}$ such that for all $n \geq N: \quad \exists \tilde{I}_{n} \subset I_{n}=\left\{1, \ldots, m_{n}\right\}$ such that $\# \tilde{I}_{n}$ $=m$ and

$$
\Omega_{j}^{n} \stackrel{d_{L_{1}}}{\longrightarrow} \Omega_{j} \quad \forall j \in \tilde{I}_{n}
$$

2. $\mu\left(D \backslash \cup_{j \in \tilde{I}_{n}} \Omega_{j}^{n}\right) \rightarrow 0$ as $n \rightarrow \infty$.

REMARK 3.2. In order to simplify notation we remark that - after a possible necessary reordering - we may write

$$
\boldsymbol{\Omega}^{n} \rightarrow \boldsymbol{\Omega} \quad: \Leftrightarrow \quad \exists N \in \mathbb{N}: \forall n \geq N:\left\{\begin{array}{lll}
\Omega_{i}^{n} \stackrel{d_{L_{1}}}{\longrightarrow} \Omega_{i} & \forall i \leq m & \text { and } n \rightarrow \infty \\
\mu\left(\Omega_{i}^{n}\right) \rightarrow 0 & \forall i>m & \text { and } n \rightarrow \infty .
\end{array}\right.
$$

To prove convergence of the minimizers of the Mumford-Shah type functional as the noise level tends to zero we need the following concept of convergence.

Definition 3.3. A sequence $\left(\boldsymbol{\Omega}^{n}, \mathbf{f}^{n}\right)_{n \in \mathbb{N}} \subset \mathcal{G}_{M}(D) \times \mathbb{R}^{m_{\boldsymbol{\Omega}}}$ converges to $(\boldsymbol{\Omega}, \mathbf{f}) \in$ $\mathcal{G}_{M}(D) \times \mathbb{R}^{m_{\boldsymbol{\Omega}}}\left(\right.$ or $\left(\boldsymbol{\Omega}^{n}, \mathbf{f}^{n}\right) \rightarrow(\boldsymbol{\Omega}, \mathbf{f})$ in short notation $)$, if

1. $\boldsymbol{\Omega}^{n} \rightarrow \boldsymbol{\Omega}$ in the sense of Definition 3.1.

2. The subsequence $\left(\mathbf{f}_{\tilde{I}_{n}}^{n}\right)_{n \in \mathbb{N}}, \tilde{I}_{n}$ acc. to Definition 3.1, fulfills

$$
\mathbf{f}_{\tilde{I}_{n}}^{n} \rightarrow \mathbf{f} .
$$

3. Coefficients related to vanishing elements of partitions stay bounded.

To guarantee that the third condition is fulfilled by the minimizers of the MumfordShah type functional the second penalty term on the norm of the coefficients is needed. Otherwise, the use of the reduced functional does not exclude the case of a sequence of partitions where at least one area shrinks to zero while the associated coefficient grows unboundedly in such a way, that the corresponding function $f$ is still in $L_{2}$. As an example, let $j$ be arbitrary but fixed with $\mu\left(\Omega_{j}^{n}\right) \sim \frac{1}{n}$. Then, we have $\left\|\mathrm{f}_{j}^{n} \chi_{\Omega_{j}^{n}}\right\|_{L_{2}}^{2} \leq\left|\mathrm{f}_{j}^{n}\right|^{2} \mu\left(\Omega_{j}^{n}\right)$, and though $\mathrm{f}_{j}^{n} \sim \sqrt{n}$ the $L_{2}$-norm of $f$ stays bounded.

Please note that in the approach of [32] the additional penalty term was not needed because vanishing sets were excluded by the problem setting. Furthermore, the results in [32] are valid only for operators that are injective on the set of piecewise constant functions. In our approach, we do not need neither of these two additional 
conditions: sets are allowed to vanish in the limit and the operator might have a non-trivial nullspace on the set of piecewise constant functions.

For sets with finite perimeter we have the following compactness [11] and lower semicontinuity result [1].

Proposition 3.4 (Compactness of finite perimeter sets in $L_{1}$ ). Let $D \subset \mathbb{R}^{d}$ be a bounded open domain with Lipschitz boundary $\partial D$. Let $\left\{\Omega_{n}\right\}_{n \in \mathbb{N}}$ be a sequence of measurable sets with uniformly bounded perimeter, i.e., $p_{D}\left(\Omega_{n}\right)=\left|\partial_{M} \Omega_{n}\right| \leq C$ for all $n \in \mathbb{N}$. Then there exists a subsequence of $\left\{\Omega_{n}\right\}$ that converges towards a measurable set $\Omega$ with respect to the metric $d_{L_{1}}$.

Proposition 3.5 (Lower semi continuity of perimeter in $L_{1}$ ). The perimeter mapping $\Omega \mapsto\left|\partial_{M} \Omega\right|$ is lower semi-continuous on the set of sets with finite perimeter with respect to the metric $d_{L_{1}}$, i.e., for any sequence $\left\{\Omega_{n}\right\}_{n \in \mathbb{N}}$ of sets with finite perimeter the implication

$$
\Omega_{n} \stackrel{d_{L_{1}}}{\longrightarrow} \Omega \Rightarrow\left|\partial_{M} \Omega\right| \leq \liminf \left|\partial_{M} \Omega_{n}\right|
$$

holds.

We now extend the compactness result to partitions with finite perimeter. A similar result was shown in [32, Proposition 5]. However, as explained above, in [32] the notion of partition is defined slightly different and hence the compactness results of [32] is for a sequence of partitions in which each sequence member as well as the limiting partition consists of the same number of sets.

Proposition 3.6 (Compactness of partitions with finite perimeter). Let $\left(\boldsymbol{\Omega}^{n}\right)_{n \in \mathbb{N}} \subset$ $\mathcal{G}_{M}(D)$ be a sequence of partitions of $D$ with uniformly bounded perimeter, i.e., there exists a constant $C>0$ such that $|\partial \boldsymbol{\Omega}|:=\sum_{i=1}^{m_{n}} p_{D}\left(\Omega_{i}^{n}\right)<C$ for all $n \in \mathbb{N}$. Then there exists a partition $\tilde{\boldsymbol{\Omega}}=\left(\tilde{\Omega}_{i}\right)_{i=1}^{\tilde{m}}$ and a subsequence of $\left(\boldsymbol{\Omega}^{n}\right)_{n \in \mathbb{N}}$, again denoted by $\left(\boldsymbol{\Omega}^{n}\right)_{n \in \mathbb{N}}$, such that

$$
\Omega^{n} \rightarrow \tilde{\Omega}
$$

in the sense of Definition 3.1.

Proof. The proof is a slight modification of [32, Proof of Propostion 5] and is given here for completeness. With $m_{n}$ we denote the number of sets in the partition $\boldsymbol{\Omega}^{n}$. As $\boldsymbol{\Omega}^{n} \in \mathcal{G}_{M}(D)$ it is $1 \leq m_{n} \leq M$ for all $n \in \mathbb{N}$. Let $m \in[1, M]$ be an arbitrary but fixed accumulation point of the sequence $\left(m_{n}\right)_{n \in \mathbb{N}}$. We consider the subsequence of partitions that consists of $m$ sets. The subsequence is again denoted by $\left(\boldsymbol{\Omega}^{n}\right)_{n \in \mathbb{N}}$.

By the compactness of finite perimeter sets, Proposition 3.4, there exists a subsequence $\left(\Omega_{1}^{1, n_{k}}\right)_{n_{k} \in \mathbb{N}}$ of $\left(\Omega_{1}^{n}\right)_{n \in \mathbb{N}}$ and a set $\tilde{\Omega}_{1}$ such that $\Omega_{1}^{1, n_{k}} \stackrel{d_{L_{1}}}{\longrightarrow} \tilde{\Omega}_{1}$ for $n_{k} \rightarrow \infty$. By the lower-semicontinuity of the perimeter map, Proposition 3.5 , we have $p_{D}\left(\tilde{\Omega}_{1}\right)<\infty$ and the limiting set $\tilde{\Omega}_{1}$ is a Caccioppoli set. We denote the subsequence $\left(\Omega_{1}^{1, n_{k}}\right)_{n_{k} \in \mathbb{N}}$ by $\left(\Omega_{1}^{1, n}\right)_{n \in \mathbb{N}}$ and the corresponding (sub)sequence of partitions by $\left(\Omega^{1, n}\right)_{n \in \mathbb{N}}$.

Set $\left(\boldsymbol{\Omega}^{0, n}\right)_{n \in \mathbb{N}}:=\left(\boldsymbol{\Omega}^{n}\right)_{n \in \mathbb{N}}$. Now, assume that for $k$ with $1 \leq k<m$ we have constructed subsequences $\left(\boldsymbol{\Omega}^{k, n}\right)_{n \in \mathbb{N}}$ of $\left(\boldsymbol{\Omega}^{k-1, n}\right)_{n \in \mathbb{N}}$ and Caccioppoli sets $\tilde{\Omega}_{k}$ such that $\Omega_{j}^{k, n} \stackrel{d_{L_{1}}}{\longrightarrow} \tilde{\Omega}_{j}$ for $n \rightarrow \infty$ and $1 \leq j \leq k$.

For the sequence of sets $\left(\Omega_{k+1}^{k, n}\right)_{n \in \mathbb{N}}$ we have $p_{D}\left(\Omega_{k+1}^{k, n}\right) \leq\left|\partial \boldsymbol{\Omega}^{k, n}\right| \leq\left|\partial \boldsymbol{\Omega}^{n}\right|$ for all $n$ and by the compactness of finite perimeter sets there exists another subsequence $\left(\boldsymbol{\Omega}^{k+1, n}\right)_{n \in \mathbb{N}}$ and a Caccioppoli set $\tilde{\Omega}_{k+1}$ such that $\Omega_{k+1}^{k+1, n} \stackrel{d_{L_{1}}}{\longrightarrow} \tilde{\Omega}_{k+1}$ for $n \rightarrow \infty$. For $1 \leq j \leq k$ the subsequence $\left(\Omega_{j}^{k+1, n}\right)_{n \in \mathbb{N}}$ inherits the limit from $\left(\Omega_{j}^{k, n}\right)_{n \in \mathbb{N}}$, i.e., we have $\Omega_{j}^{k+1, n} \stackrel{d_{L_{1}}}{\longrightarrow} \tilde{\Omega}_{j}$. 
Induction with respect to $k$ finally leads to a subsequence $\left(\boldsymbol{\Omega}^{m, n}\right)_{n \in \mathbb{N}}$, again denoted by $\left(\boldsymbol{\Omega}^{n}\right)_{n \in \mathbb{N}}$ and $m$ sets $\tilde{\Omega}_{i}, i=1, \ldots, m$, such that $\Omega_{i}^{n} \stackrel{d_{L_{1}}}{\longrightarrow} \tilde{\Omega}_{i}$ for all $1 \leq i \leq m$ and $n \rightarrow \infty$. Because of

$$
\sum_{i=1}^{m} \chi_{\tilde{\Omega}_{i}}=\lim _{n \rightarrow \infty} \sum_{i=1}^{m} \chi_{\Omega_{i}^{m, n}}=\lim _{n \rightarrow \infty} \chi_{D}=\chi_{D}
$$

there exists at least one $j \in 1, \ldots, m$ such that $\mu\left(\tilde{\Omega}_{j}\right)>0$. Denoting the number of sets with non-zero Lebesgue measure by $\tilde{m}$ we have that, after a possible renumbering of the limiting sets, $\tilde{\boldsymbol{\Omega}}:=\left(\tilde{\Omega}_{i}\right)_{i=1}^{\tilde{m}}$ is a partition of $D$ and $\boldsymbol{\Omega}^{n} \rightarrow \tilde{\boldsymbol{\Omega}}$ in the sense of Definition 3.1.

4. Optimal coefficients for a given geometry. In this section we compute optimal coefficients of the Mumford-Shah type functional for a fixed geometry and analyze their behavior for convergent sequences of partitions.

First we want to determine the coefficients that minimize the defect for a given (fixed) partition $\boldsymbol{\Omega}=\left(\Omega_{i}\right)_{i=1}^{m}$.

Proposition 4.1. Let $\boldsymbol{\Omega}$ be a fixed, given partition, $\beta>0$ and $g^{\delta} \in L_{2}$ and let $f=F(\boldsymbol{\Omega}, \mathbf{f})=\sum_{i=1}^{m} f_{i} \chi_{\Omega_{i}}$ be the unknown reconstruction. Then the (unique) minimizer of the functional

$$
\min _{\mathbf{f} \in \mathbb{R}^{m}}\left\|K F(\boldsymbol{\Omega}, \mathbf{f})-g^{\delta}\right\|_{L_{2}}^{2}+\beta\|\mathbf{f}\|_{\ell_{2}}^{2}
$$

exists and is given by the solution $\mathbf{f}=\left(\mathrm{f}_{i}\right)_{i=1}^{m}$ of the matrix-vector system

$$
\mathbf{g}^{\delta}=\mathbf{K} \mathbf{f}
$$

where $\mathbf{g}^{\delta}=\left(g_{i}^{\delta}\right)_{i=1}^{m}$ and $\mathbf{K}=\left(k_{i j}\right)_{i, j=1}^{m}$ with

$$
\begin{aligned}
g_{i}^{\delta} & =\left\langle\chi_{\Omega_{i}}, K^{*} g^{\delta}\right\rangle \\
k_{i j} & =\left\langle K \chi_{\Omega_{i}}, K \chi_{\Omega_{j}}\right\rangle+\beta \delta_{i j} .
\end{aligned}
$$

Proof. As the geometry is fix, we identify functions $h \in V_{\Omega}:=\operatorname{span}\left\{\chi_{\Omega_{i}}\right.$ : $i=1, \ldots m\} \subset L_{2}(D)$ with their coefficient vector $\mathbf{h}=\left(h_{i}\right)_{i=1}^{m} \in \mathbb{R}^{m}$. For the optimization we use the operator

$$
\mathcal{K}: \mathbb{R}^{m} \rightarrow Y, \quad \mathbf{h} \mapsto \sum_{i=1}^{m} h_{i} K \chi_{\Omega_{i}}
$$

and its adjoint

$$
\mathcal{K}^{*}: Y \rightarrow \mathbb{R}^{m}, \quad g \mapsto\left(\left\langle\chi_{\Omega_{i}}, K^{*} g\right\rangle\right)_{i=1, . ., m} .
$$

With $K h=\mathcal{K} \mathbf{h}$ the necessary condition for a minimizer of the functional (4.1) is given by

$$
\left\langle\mathcal{K}^{*}\left(\mathcal{K} \mathbf{f}-g^{\delta}\right), \mathbf{h}\right\rangle_{\ell_{2}}+\beta\langle\mathbf{f}, \mathbf{h}\rangle_{\ell_{2}}=0 \quad \forall \mathbf{h} \in \mathbb{R}^{m} .
$$

Equation (4.4) holds if

$$
\mathcal{K}^{*} \mathcal{K} \mathbf{f}+\beta \mathbf{f}=\mathcal{K}^{*} g^{\delta}
$$


or

$$
\left(\mathcal{K}^{*} \mathcal{K} \mathbf{f}+\beta \mathbf{f}\right)_{i}=\left(\mathcal{K}^{*} g^{\delta}\right)_{i} \quad \forall i=1, \ldots, m
$$

The assertion follows with

$$
\left(\mathcal{K}^{*} g^{\delta}\right)_{i}=\left\langle\chi_{\Omega_{i}}, K^{*} g^{\delta}\right\rangle \stackrel{(4.3 \mathrm{a})}{=} g_{i}^{\delta}
$$

and

$$
\begin{aligned}
\left(\mathcal{K}^{*} \mathcal{K} \mathbf{f}+\beta \mathbf{f}\right)_{i} & =\left(\mathcal{K}^{*} \mathcal{K} \mathbf{f}\right)_{i}+\beta(\mathbf{f})_{i} \\
& =\left\langle\chi_{\Omega_{i}}, K^{*} \mathcal{K} \mathbf{f}\right\rangle+\beta \mathrm{f}_{i} \\
& =\left\langle K \chi_{\Omega_{i}}, \sum \mathrm{f}_{j} K \chi_{\Omega_{j}}\right\rangle_{Y}+\beta \mathrm{f}_{i} \\
& =\sum_{j=1}^{m} k_{i j} \mathrm{f}_{j}
\end{aligned}
$$

with $k_{i j}$ according to (4.3b). Existence and uniqueness of a solution of the linear system is guaranteed by Lemma 4.2 and $\beta>0$.

Lemma 4.2. For $\beta>0$ the matrix $\mathbf{K}$ in (4.2) is positive definite. Hence, a unique solution to the linear system exists.

Proof. Let $h=\sum_{i=1}^{m} h_{i} \chi_{\Omega_{i}} \neq 0$. For $i=1, \ldots, m$ we have $\mu\left(\Omega_{i}\right)>0$ which implies $\mathbf{h} \neq 0$. It is

$$
\begin{aligned}
\langle\mathbf{h}, \mathbf{K h}\rangle_{\mathbb{R}^{m}} & =\sum_{i, j=1}^{m} h_{i} k_{i j} h_{j} \\
& =\sum_{i, j=1}^{m} h_{i}\left\langle K \chi_{\Omega_{i}}, K \chi_{\Omega_{j}}\right\rangle_{Y} h_{j}+\beta \sum_{i=1}^{m} h_{i}^{2} \\
& =\langle K h, K h\rangle_{Y}+\beta\|\mathbf{h}\|_{\ell_{2}}^{2} \\
& =\|K h\|_{Y}^{2}+\beta\|\mathbf{h}\|_{\ell_{2}}^{2} \\
& >0 \quad \text { as } \beta>0, \mathbf{h} \neq 0 .
\end{aligned}
$$

Hence, for $\beta>0$ the matrix $\mathbf{K}$ is positive definite. $\square$

Now, we aim at a stability result for the solution to (4.1) under perturbations of the geometry. Assume that we are given a sequence of partitions $\left(\boldsymbol{\Omega}^{n}\right)_{n \in \mathbb{N}}$ with $\boldsymbol{\Omega}^{n}=\left(\Omega_{i}^{n}\right)_{i=1}^{m_{n}}$ and a partition $\boldsymbol{\Omega}=\left(\Omega_{i}\right)_{i=1}^{m}$ such that $\boldsymbol{\Omega}^{n} \rightarrow \boldsymbol{\Omega}$ for $n \rightarrow \infty$ in the sense of Definition 3.1. The number of sets in the partitions $\boldsymbol{\Omega}^{n}$ and $\boldsymbol{\Omega}$ is denoted by $m_{n}$ and $m$, respectively. Hence, the according linear systems in Proposition 4.1 have dimension $m_{n} \times m_{n}$ and $m \times m$, respectively. The number of sets in $\boldsymbol{\Omega}^{n}$ and $\boldsymbol{\Omega}$ does not necessarily coincide even for large $n$. However, as $\boldsymbol{\Omega}^{n} \rightarrow \boldsymbol{\Omega}$ there is an $N \in \mathbb{N}$ such that

$$
\forall n \geq N: m_{n} \geq m \text {. }
$$

According to Remark 3.2, for each partition $\boldsymbol{\Omega}^{n}$ with $n \geq N$, we can reorder the indices $1, \ldots, m_{n}$ such that

$$
\Omega_{i}^{n} \stackrel{d_{L_{1}}}{\longrightarrow} \Omega_{i} \text { for } i=1, \ldots, m \text { and } \mu\left(\Omega_{i}^{n}\right) \rightarrow 0 \text { for } i>m .
$$


For each partition $\boldsymbol{\Omega}^{n}, \boldsymbol{\Omega}$ we compute the solution to (4.1) as solution of the equations $\mathbf{K}^{n} \mathbf{f}^{n}=\mathbf{g}^{\delta, n}$ and $\mathbf{K f}=\mathbf{g}^{\delta}$. The notation $\mathbf{K}^{n}$ is used for the matrix that belongs to the $n$th partition, the upper index $n$ is not to be misunderstood as an exponent. According to the reordering of the indices, the system matrices $\mathbf{K}^{n}$ can be written as

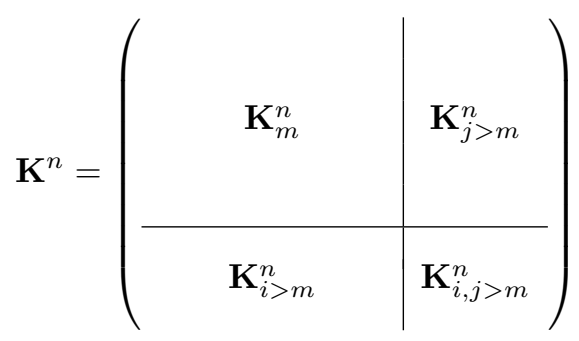

with $\mathbf{K}_{m}^{n} \in \mathbb{R}^{m \times m}, \mathbf{K}_{j>m}^{n} \in \mathbb{R}^{m \times\left(m_{n}-m\right)}, \mathbf{K}_{i>m}^{n} \in \mathbb{R}^{\left(m_{n}-m\right) \times m}$ and $\mathbf{K}_{i, j>m}^{n} \in$ $\mathbb{R}^{\left(m_{n}-m\right) \times\left(m_{n}-m\right)}$. Following the lines of the proof of Lemma 4.2 we see that the reordering does not change the positive definiteness.

The data vector $\mathbf{g}^{\delta, n}$ and the coefficient vector $\mathbf{f}^{n}$ are reordered analogously,

$$
\mathbf{g}^{\delta, n}=\left(\begin{array}{c}
\mathbf{g}_{m}^{\delta, n} \\
\hline \mathbf{g}_{i>m}^{\delta, n}
\end{array}\right) \quad \text { and } \quad \mathbf{f}^{n}=\left(\begin{array}{c}
\mathbf{f}_{m}^{n} \\
\hline \mathbf{f}_{i>m}^{n}
\end{array}\right)
$$

with $\mathbf{g}_{m}^{n}, \mathbf{f}_{m}^{n} \in \mathbb{R}^{m}$ and $\mathbf{g}_{i>m}^{n}, \mathbf{f}_{i>m}^{n} \in \mathbb{R}^{\left(m_{n}-m\right)}$.

The following approximation result holds for the upper left part $\mathbf{K}_{m}^{n}$ of the matrix $\mathbf{K}^{n}$.

Proposition 4.3. The elements of the matrices $\mathbf{K}$ and $\mathbf{K}_{m}^{n}$ fulfill

$$
\left|k_{i j}-k_{i j}^{n}\right| \leq \varepsilon_{n}(i, j)
$$

where $\varepsilon_{n}(i, j)$ is defined by

$$
\varepsilon_{n}(i, j)=\sqrt{\mu(D)}\left\|K^{*} K\right\|_{2}\left(\sqrt{d_{L_{1}}\left(\Omega_{i}^{n}, \Omega_{i}\right)}+\sqrt{d_{L_{1}}\left(\Omega_{j}^{n}, \Omega_{j}\right)}\right) .
$$

Moreover,

$$
\varepsilon_{n}(i, j) \rightarrow 0 \text { as } n \rightarrow \infty .
$$

Proof. It is

$$
\begin{aligned}
\left|k_{i j}^{n}-k_{i j}\right| & =\left|\left\langle K \chi_{\Omega_{i}^{n}}, K \chi_{\Omega_{j}^{n}}\right\rangle-\left\langle K \chi_{\Omega_{i}}, K \chi_{\Omega_{j}}\right\rangle\right| \\
& \leq \mid\left\langle\chi_{\Omega_{i}^{n}}, K^{*} K\left(\chi_{\Omega_{j}^{n}}-\chi_{\Omega_{j}}\right\rangle|+|\left\langle\chi_{\Omega_{j}}, K^{*} K\left(\chi_{\Omega_{i}^{n}}-\chi_{\Omega_{i}}\right\rangle\right|\right. \\
& \leq\left\|K^{*} K\right\|\left(\sqrt{\mu\left(\Omega_{i}^{n}\right)}\left\|\chi_{\Omega_{j}^{n}}-\chi_{\Omega_{j}}\right\|_{2}+\sqrt{\mu\left(\Omega_{j}\right)}\left\|\chi_{\Omega_{i}^{n}}-\chi_{\Omega_{i}}\right\|_{2}\right), \\
& \leq \sqrt{\mu(D)}\left\|K^{*} K\right\|\left(\left\|\chi_{\Omega_{j}^{n}}-\chi_{\Omega_{j}}\right\|_{2}+\left\|\chi_{\Omega_{i}^{n}}-\chi_{\Omega_{i}}\right\|_{2}\right),
\end{aligned}
$$


where we have used that $\left\|\chi_{D}\right\|_{2}=\sqrt{\mu(D)}$ and $\mu\left(\Omega_{j}^{n}\right), \mu\left(\Omega_{j}\right) \leq \mu(D)$. Assertion (4.7) follows with $\left\|\chi_{\Omega_{i, n}}-\chi_{\Omega_{i}}\right\|_{2}=\sqrt{d_{L_{1}}\left(\Omega_{i}^{n}, \Omega_{i}\right)}$.

The convergence of the sets, $\Omega_{i}^{n} \stackrel{d_{L_{1}}}{\longrightarrow} \Omega_{i}$, implies $\varepsilon_{n}(i, j) \rightarrow 0$ as $n \rightarrow \infty$.

COROLlary 4.4. The sequence $\left(\mathbf{K}_{m}^{n}\right)_{n \in \mathbb{N}}$ of the upperleft parts of the matrices $\mathbf{K}^{n}$ converges (in norm) to the matrix $K$.

Proof. This is an immediate consequence of Proposition 4.3 and the finite dimension of the matrices. $\square$

LEMMA 4.5. The elements of the matrix $\mathbf{K}_{j>m}^{n}$ fulfill

$$
k_{i j}^{n} \rightarrow 0 \quad \text { as } n \rightarrow \infty
$$

Proof. Note that $\mathbf{K}_{j>m}^{n}=\left(k_{i j}^{n}\right)_{i j}$ with $i=1, \ldots, m$ and $j>m$. Hence the term $\beta \delta_{i j}$ in (4.3b) always vanishes and it is

$$
\begin{aligned}
\left|k_{i j}^{n}\right| & =\left|\left\langle K \chi_{\Omega_{i}^{n}}, K \chi_{\Omega_{j}^{n}}\right\rangle\right|=\left|\left\langle K^{*} K \chi_{\Omega_{i}^{n}}, \chi_{\Omega_{j}^{n}}\right\rangle\right| \\
& \leq\left\|K^{*} K\right\| \sqrt{\mu\left(\Omega_{j}^{n}\right)} \sqrt{\mu\left(\Omega_{i}^{n}\right)} \rightarrow 0
\end{aligned}
$$

as $\mu\left(\Omega_{j}^{n}\right) \rightarrow 0$ for $j>m$ and $\mu\left(\Omega_{i}^{n}\right) \leq \mu(D) \forall i$.

The other two submatrices also converge to zero. However, this is not important as they do not appear in our future analysis. A similar approximation result as for the matrices $\mathbf{K}_{m}^{n}$ and $\mathbf{K}$ also holds for the data vectors $\mathbf{g}_{m}^{\delta, n}$ and $\mathbf{g}^{\delta}$.

Lemma 4.6. Let $K: L_{2}(D) \rightarrow L_{2}\left(D_{2}\right)$ be a bounded linear operator. For $\mathbf{g}_{m}^{\delta, n}$ and $\mathbf{g}^{\delta}$ the estimate

$$
\left|g_{i}^{\delta, n}-g_{i}^{\delta}\right| \leq C \cdot \sqrt{d_{L_{1}}\left(\Omega_{i}^{n}, \Omega_{i}\right)}
$$

holds for all $1 \leq i \leq m$ with some constant $C>0$.

Proof. With (4.3a) it is

$$
\left|g_{i}^{\delta, n}-g_{i}^{\delta}\right|=\left|\left\langle\chi_{\Omega_{i}^{n}}-\chi_{\Omega_{i}}, K^{*} g^{\delta}\right\rangle_{L_{2}}\right| \leq\left\|\chi_{\Omega_{i}^{n}}-\chi_{\Omega_{i}}\right\|_{L_{2}}\left\|K^{*} g^{\delta}\right\|_{L_{2}} .
$$

With the help of these auxiliary results we now prove convergence of the sequence of coefficient vectors.

Proposition 4.7. Let $\left(\boldsymbol{\Omega}^{n}\right)_{n \in \mathbb{N}}$ and $\boldsymbol{\Omega}$ be partitions with $\boldsymbol{\Omega}^{n}=\left(\Omega_{i}^{n}\right)_{i=1, \ldots, m_{n}}$ and $\boldsymbol{\Omega}=\left(\Omega_{i}\right)_{i=1, \ldots, m}$; assume $\boldsymbol{\Omega}^{n} \rightarrow \boldsymbol{\Omega}$ as $n \rightarrow \infty$. Let $\beta>0$ and $\mathbf{f}^{n}=\left(\mathbf{f}_{m}^{n}, \mathbf{f}_{i>m}^{n}\right)$ and $\mathbf{f}$ be the solutions to $\mathbf{K}^{n} \mathbf{f}^{n}=\mathbf{g}^{\delta, n}$ and $\mathbf{K f}=\mathbf{g}^{\delta}$. Assume further that $\left\|\mathbf{f}^{n}\right\| \leq C<\infty$. Then, the sequence $\left(\mathbf{f}_{m}^{n}\right)_{n \in \mathbb{N}}$ converges to $\mathbf{f}$,

$$
\mathbf{f}_{m}^{n} \rightarrow \mathbf{f} \quad \text { as } n \rightarrow \infty
$$

Proof. For the convergence of the first $m$ coefficients we use a standard perturbation argument from linear algebra (see Kress [20], Theorem 10.1, page 164). As $\beta>0$ we know from Lemma 4.2 that the matrix $\mathbf{K}$ is positive definite and hence invertible. Since $\mathbf{K}_{m}^{n}$ and $\mathbf{K}$ have the same dimensions and since $\mathbf{K}_{m}^{n} \rightarrow \mathbf{K}$ as $n \rightarrow \infty$ due to Corollary 4.4 we can choose $n$ large enough such that $\left\|\mathbf{K}^{-1}\left(\mathbf{K}_{m}^{n}-\mathbf{K}\right)\right\|<1$ where $\|\cdot\|$ is an arbitrary matrix norm induced by a vector norm on $\mathbb{R}^{m}$. Using a 
Neumann series argument it is apparent that $I+\mathbf{K}^{-1}\left(\mathbf{K}_{m}^{n}-\mathbf{K}\right)$ is invertible. We have (compare (4.5) and (4.6))

$$
\begin{aligned}
\mathbf{g}_{m}^{\delta, n}-\mathbf{g}^{\delta} & =\mathbf{K}_{m}^{n} \mathbf{f}_{m}^{n}+\mathbf{K}_{j>m}^{n} \mathbf{f}_{i>m}^{n}-\mathbf{K} \mathbf{f} \\
& =\left(\mathbf{K}+\left(\mathbf{K}_{m}^{n}-\mathbf{K}\right)\right)\left(\mathbf{f}_{m}^{n}-\mathbf{f}\right)-\left(\mathbf{K}_{m}^{n}-\mathbf{K}\right) \mathbf{f}+\mathbf{K}_{j>m}^{n} \mathbf{f}_{i>m}^{n} .
\end{aligned}
$$

Consequently

$$
\mathbf{K}\left(I+\mathbf{K}^{-1}\left(\mathbf{K}_{m}^{n}-\mathbf{K}\right)\right)\left(\mathbf{f}_{m}^{n}-\mathbf{f}\right)=\mathbf{g}_{m}^{\delta, n}-\mathbf{g}^{\delta}+\left(\mathbf{K}_{m}^{n}-\mathbf{K}\right) \mathbf{f}-\mathbf{K}_{j>m}^{n} \mathbf{f}_{i>m}^{n}
$$

and therefore

$$
\mathbf{f}_{m}^{n}-\mathbf{f}=\left(I+\mathbf{K}^{-1}\left(\mathbf{K}_{m}^{n}-\mathbf{K}\right)\right)^{-1} \mathbf{K}^{-1}\left(\mathbf{g}_{m}^{\delta, n}-\mathbf{g}^{\delta}+\left(\mathbf{K}_{m}^{n}-\mathbf{K}\right) \mathbf{f}-\mathbf{K}_{j>m}^{n} \mathbf{f}_{i>m}^{n}\right)
$$

or

$$
\left\|\mathbf{f}_{m}^{n}-\mathbf{f}\right\| \leq \frac{\left\|\mathbf{K}^{-1}\right\|}{1-\left\|\mathbf{K}^{-1}\left(\mathbf{K}_{m}^{n}-\mathbf{K}\right)\right\|}\left(\left\|\mathbf{g}_{m}^{\delta, n}-\mathbf{g}^{\delta}\right\|+\left\|\mathbf{K}_{m}^{n}-\mathbf{K}\right\|\|\mathbf{f}\|+\left\|\mathbf{K}_{j>m}^{n}\right\|\left\|\mathbf{f}_{i>m}^{n}\right\|\right) .
$$

Here we used that $\left\|(I+A)^{-1}\right\| \leq \frac{1}{1-\|A\|}$ for every quadratic matrix $A$ with $\|A\|<1$. Application of Corollary 4.4, Lemma 4.5 and Lemma 4.6 together with the boundedness of the $\mathbf{f}^{n}$ shows that the right-hand side of this inequality converges to zero as $n \rightarrow \infty$.

Corollary 4.8. Under the conditions of Proposition 4.7 and setting $f=$ $F(\boldsymbol{\Omega}, \mathbf{f}), f^{n}=F\left(\boldsymbol{\Omega}^{n}, \mathbf{f}^{n}\right)$ we have

$$
f^{n} \stackrel{L_{2}}{\longrightarrow} f
$$

Proof. We have $\left\|f^{n}\right\| \leq C<\infty$ and hence $\left|\mathrm{f}_{i}\right| \leq C$ for all $i=1, \ldots, m_{n}$. Furthermore, it is $\mu\left(\Omega_{i}^{n}\right) \leq \mu(D)$ for all $i=1, \ldots, m_{n}$. We get

$$
\begin{aligned}
\left\|f-f^{n}\right\|_{L_{2}} & =\left\|\sum_{i=1}^{m} \mathrm{f}_{i} \chi_{\Omega_{i}}-\sum_{i=1}^{m_{n}} \mathrm{f}_{i}^{n} \chi_{\Omega_{i}^{n}}\right\|_{L_{2}} \\
& \leq\left\|\sum_{i=1}^{m} \mathrm{f}_{i}^{n}\left(\chi_{\Omega_{i}^{n}}-\chi_{\Omega_{i}}\right)\right\|_{L_{2}}+\left\|\sum_{i=1}^{m}\left(\mathrm{f}_{i}^{n}-\mathrm{f}_{i}\right) \chi_{\Omega_{i}}\right\|_{L_{2}}+\left\|\sum_{i=m+1}^{m_{n}} \mathrm{f}_{i}^{n} \chi_{\Omega_{i}^{n}}\right\|_{L_{2}} \\
& \leq C \sum_{i=1}^{m} d_{L_{1}}\left(\Omega_{i}^{n}, \Omega_{i}\right)+\sqrt{\mu(D)} \sum_{i=1}^{m}\left|\mathrm{f}_{i}^{n}-\mathrm{f}_{i}\right|+C \sum_{i=m+1}^{m_{n}} \sqrt{\mu\left(\Omega_{i}^{n}\right)} .
\end{aligned}
$$

The assertion follows with $\boldsymbol{\Omega}^{n} \rightarrow \boldsymbol{\Omega}$ and Proposition 4.7.

5. Existence, stability and regularization results. In this section we present the main properties of the reconstructions based on the described Mumford-Shah type method, namely existence of a minimizer (Theorem 5.1), stability of the minimizer with respect to error in the data (Theorem 5.3), and that the presented approach is a regularization method if equipped with a proper parameter choice rule (Theorem 5.4).

As it is frequently used in the following theorems we would like to recall the definition of convergence of a sequence $\left(\boldsymbol{\Omega}^{n}, \mathbf{f}^{n}\right)_{n \in \mathbb{N}}$ consisting of pairs of a partition and a coefficient vector. According to Definitions 3.3 and 3.1 we request that the sets $\Omega_{i}^{n}$ converge with respect to $d_{L_{1}}$. However, the associated components of the 
coefficient vectors only have to converge whenever the associated limiting set has measure greater than zero. The remaining coefficients only have to stay bounded which is always guaranteed by the norm penalty in the Mumford-Shah type functional.

TheOREM 5.1. The Mumford-Shah functional defined in (2.8) with fixed $\beta, \gamma>0$ has a (not necessarily unique) minimizer $\left(\boldsymbol{\Omega}_{\beta, \gamma}^{\delta}, \mathbf{f}_{\beta, \gamma}^{\delta}\right) \in \mathcal{G}_{M}(D) \times \mathbb{R}^{m}$ for each set of data $g^{\delta}$.

Proof. The functional $J_{\beta, \gamma}$ is bounded from below by zero and there clearly exists an argument $(\boldsymbol{\Omega}, \mathbf{f})$ such that $J_{\beta, \gamma}(\boldsymbol{\Omega}, \mathbf{f})<\infty$. Hence, there exists a minimizing sequence $\left(\tilde{\boldsymbol{\Omega}}^{n}, \tilde{\mathbf{f}}^{n}\right)$ with $J_{\beta, \gamma}\left(\tilde{\boldsymbol{\Omega}}^{n}, \tilde{\mathbf{f}}^{n}\right) \rightarrow \inf J_{\beta, \gamma}(\boldsymbol{\Omega}, \mathbf{f})$ and -after possibly extracting a subsequence- $J_{\beta, \gamma}\left(\tilde{\boldsymbol{\Omega}}^{n}, \tilde{\mathbf{f}}^{n}\right) \leq J_{\beta, \gamma}\left(\tilde{\boldsymbol{\Omega}}^{n-1}, \tilde{\mathbf{f}}^{n-1}\right)$. In particular, we have $\left|\partial \tilde{\boldsymbol{\Omega}}^{n}\right| \leq \frac{1}{\gamma} J_{\beta, \gamma}\left(\tilde{\boldsymbol{\Omega}}^{0}, \tilde{\mathbf{f}}^{0}\right)$. Hence, $\left(\tilde{\boldsymbol{\Omega}}^{n}\right)_{n \in \mathbb{N}}$ has uniformly bounded perimeter and by Proposition 3.6 there exists a subsequence of partitions $\boldsymbol{\Omega}^{n}$ of $\tilde{\boldsymbol{\Omega}}^{n}$ and a limiting partition $\boldsymbol{\Omega}$ such that $\boldsymbol{\Omega}^{n} \stackrel{d_{L_{1}}}{\longrightarrow} \boldsymbol{\Omega}$ for $n \rightarrow \infty$. Now we construct a new minimizing sequence by changing the coefficients $\tilde{\mathbf{f}}^{n}$ for the subsequence of partitions $\boldsymbol{\Omega}^{n}$. We define the vector $\mathbf{f}^{n}$ to be the solution of the linear system (4.2) with fixed geome$\operatorname{try} \boldsymbol{\Omega}^{n}$, i.e., the set of coefficients that minimizes the functional (4.1). According to Proposition 4.1 we have $J_{\beta, \gamma}\left(\boldsymbol{\Omega}^{n}, \mathbf{f}^{n}\right) \leq J_{\beta, \gamma}\left(\boldsymbol{\Omega}^{n}, \tilde{\mathbf{f}}^{n}\right)$, i.e., $\left(\boldsymbol{\Omega}^{n}, \mathbf{f}^{n}\right)$ is also a minimizing sequence for $J_{\beta, \gamma}$ and the associated coefficients are bounded. For the limiting partition $\boldsymbol{\Omega}$ we define $\mathbf{f}$ as the solution of the linear system (4.2). The vector $\mathbf{f}^{n}$ is split according to (4.6). According to Proposition 4.7, eq. (4.9), we have $\mathbf{f}_{m}^{n} \rightarrow \mathbf{f}$ where $\mathbf{f}_{m}^{n}$ denotes the reordered first $m$ coefficients of each of the coefficient vectors $\mathbf{f}^{n}$; i.e., coefficients associated to sets that do not vanish in the limit.

From Corollary 4.8 we get $f^{n}=F\left(\boldsymbol{\Omega}^{n}, \mathbf{f}^{n}\right) \stackrel{d_{L_{2}}}{\longrightarrow} F(\boldsymbol{\Omega}, \mathbf{f})=f$. By the lower semicontinuity of norm and perimeter it follows

$$
\begin{aligned}
J_{\beta, \gamma}(\boldsymbol{\Omega}, \mathbf{f}) & \leq \liminf _{n \rightarrow \infty}\left(\left\|K f^{n}-g^{\delta}\right\|_{L_{2}(D)}^{2}+\beta\left\|\mathbf{f}_{m}^{n}\right\|_{\ell_{2}}^{2}+\gamma\left|\partial \boldsymbol{\Omega}^{n}\right|\right) \\
& \leq \liminf _{n \rightarrow \infty}\left(\left\|K f^{n}-g^{\delta}\right\|_{L_{2}(D)}^{2}+\beta\left(\left\|\mathbf{f}_{m}^{n}\right\|_{\ell_{2}}^{2}+\left\|\mathbf{f}_{i>m}^{n}\right\|_{\ell_{2}}^{2}\right)+\gamma\left|\partial \boldsymbol{\Omega}^{n}\right|\right) \\
& =\liminf _{n \rightarrow \infty} J_{\beta, \gamma}\left(\boldsymbol{\Omega}^{n}, \mathbf{f}^{n}\right) \\
& =\inf _{\beta, \gamma}(\boldsymbol{\Omega}, \mathbf{f}) .
\end{aligned}
$$

The next lemma is used to show the stability of the minimizers of the MumfordShah like functional with respect to perturbations in the data.

Lemma 5.2. Let $g^{\delta_{n}} \rightarrow g^{\delta}$ and denote by $\left(\boldsymbol{\Omega}_{\beta, \gamma}^{\delta_{n}}, \mathbf{f}_{\beta, \gamma}^{\delta_{n}}\right)$ and $\left(\boldsymbol{\Omega}_{\beta, \gamma}^{\delta}, \mathbf{f}_{\beta, \gamma}^{\delta}\right)$ the minimizers of the functionals $J_{\beta, \gamma}$ with data $g^{\delta_{n}}$ and $g^{\delta}$. Then, the sequence of minimizing partitions and the sequence of minimizing coefficients are uniformly bounded, i.e., there exist constants $C_{1}, C_{2} \geq 0$ such that

$$
\begin{aligned}
\left|\partial \boldsymbol{\Omega}_{\beta, \gamma}^{\delta_{n}}\right| \leq C_{1}<\infty \quad \forall n \\
\left\|\mathbf{f}_{\beta, \gamma}^{n}\right\| \leq C_{2}<\infty \quad \forall n .
\end{aligned}
$$

The proof is analogous to the proof of [32, Lemma 13]. We present it for completeness.

Proof. Let $f_{\beta, \gamma}^{\delta_{n}}=F\left(\boldsymbol{\Omega}_{\beta, \gamma}^{\delta_{n}}, \mathbf{f}_{\beta, \gamma}^{\delta_{n}}\right)$. As $\left(\boldsymbol{\Omega}_{\beta, \gamma}^{\delta_{n}}, \mathbf{f}_{\beta, \gamma}^{\delta_{n}}\right)$ is a minimizer of $J_{\beta, \gamma}$ with data $g^{\delta_{n}}$ we have

$$
\begin{aligned}
\gamma\left|\partial \boldsymbol{\Omega}_{\beta, \gamma}^{\delta_{n}}\right| & \leq\left\|K f_{\beta, \gamma}^{\delta_{n}}-g^{\delta_{n}}\right\|_{L_{2}(D)}^{2}+\beta\left\|\mathbf{f}_{\beta, \gamma}^{\delta_{n}}\right\|_{\ell_{2}}^{2}+\gamma\left|\partial \boldsymbol{\Omega}_{\beta, \gamma}^{\delta_{n}}\right| \\
& \leq\left\|K f_{\beta, \gamma}^{\delta}-g^{\delta_{n}}\right\|_{L_{2}(D)}^{2}+\beta\left\|\mathbf{f}_{\beta, \gamma}^{\delta}\right\|_{\ell_{2}}^{2}+\gamma\left|\partial \boldsymbol{\Omega}_{\beta, \gamma}^{\delta}\right|
\end{aligned}
$$


As $\lim _{n \rightarrow \infty}\left\|K f_{\beta, \gamma}^{\delta}-g^{\delta_{n}}\right\|_{L_{2}(D)}^{2}=\left\|K f_{\beta, \gamma}^{\delta}-g^{\delta}\right\|_{L_{2}(D)}^{2}$ we conclude that $\| K f_{\beta, \gamma}^{\delta}-$ $g^{\delta_{n}} \|_{L_{2}(D)}^{2} \leq \tilde{C}$ for $n$ sufficiently large. It follows that

$$
\left|\partial \boldsymbol{\Omega}_{\beta, \gamma}^{\delta_{n}}\right| \leq \frac{\tilde{C}}{\gamma}+\frac{\beta}{\gamma}\left\|\mathbf{f}_{\beta, \gamma}^{\delta}\right\|_{\ell_{2}}^{2}+\left|\partial \boldsymbol{\Omega}_{\beta, \gamma}^{\delta}\right|
$$

independent of $n$. An analogous computation yields

$$
\left\|\mathbf{f}_{\beta, \gamma}^{\delta_{n}}\right\| \leq \frac{\tilde{C}}{\beta}+\left\|\mathbf{f}_{\beta, \gamma}^{\delta}\right\|_{\ell_{2}}^{2}+\frac{\gamma}{\beta}\left|\partial \boldsymbol{\Omega}_{\beta, \gamma}^{\delta}\right|
$$

TheOREM 5.3. Let $g^{\delta_{n}} \rightarrow g^{\delta}$ in $L_{2}$ and denote by $\left(\boldsymbol{\Omega}_{\beta, \gamma}^{\delta_{n}}, \mathbf{f}_{\beta, \gamma}^{\delta_{n}}\right)$ a sequence of minimizers of the functionals $J_{\beta, \gamma}$ with data $g^{\delta_{n}}$. Then there exists a subsequence of $\left(\boldsymbol{\Omega}_{\beta, \gamma}^{\delta_{n}}, \mathbf{f}_{\beta, \gamma}^{\delta_{n}}\right)$, again denoted by $\left(\boldsymbol{\Omega}_{\beta, \gamma}^{\delta_{n}}, \mathbf{f}_{\beta, \gamma}^{\delta_{n}}\right)$, such that

$$
\left(\boldsymbol{\Omega}_{\beta, \gamma}^{\delta_{n}}, \mathbf{f}_{\beta, \gamma}^{\delta_{n}}\right) \rightarrow\left(\boldsymbol{\Omega}_{\beta, \gamma}^{\delta}, \mathbf{f}_{\beta, \gamma}^{\delta}\right)
$$

in the sense of Definition 3.3 and $\left(\boldsymbol{\Omega}_{\beta, \gamma}^{\delta}, \mathbf{f}_{\beta, \gamma}^{\delta}\right)$ is a minimizer of $J_{\beta, \gamma}$ with data $g^{\delta}$.

Proof. According to Theorem 5.1, minimizers $\left(\boldsymbol{\Omega}_{\beta, \gamma}^{\delta_{n}}, \mathbf{f}_{\beta, \gamma}^{\delta_{n}}\right)$ of the functional $J_{\beta, \gamma}$ with data $g^{\delta_{n}}$ exists. By Lemma 5.2 we have that the perimeter of the partitions and the norm of the coefficients are uniformly bounded, i.e., $\left|\partial \boldsymbol{\Omega}_{\beta, \gamma}^{\delta_{n}}\right| \leq C_{1}$ and $\left\|\mathbf{f}_{\beta, \gamma}^{\delta_{n}}\right\| \leq C_{2}$.

Analogous to the proof of Theorem 5.1, by using Proposition 4.7, there exists a subsequence of $\left(\boldsymbol{\Omega}_{\beta, \gamma}^{\delta_{n}}, \mathbf{f}_{\beta, \gamma}^{\delta_{n}}\right)$, for simplicity again denoted by $\left(\boldsymbol{\Omega}_{\beta, \gamma}^{\delta_{n}}, \mathbf{f}_{\beta, \gamma}^{\delta_{n}}\right)$, and an element $(\tilde{\boldsymbol{\Omega}}, \tilde{\mathbf{f}}) \in \mathcal{G}_{M}(D) \times \mathbb{R}^{m}$ such that

$$
\boldsymbol{\Omega}_{\beta, \gamma}^{\delta_{n}} \rightarrow \tilde{\boldsymbol{\Omega}} \text { for } n \rightarrow \infty \text { and }\left(\mathbf{f}_{\beta, \gamma}^{\delta_{n}}\right)_{m} \rightarrow \tilde{\mathbf{f}} \text { for } n \rightarrow \infty,
$$

with a possible reordering of the vector as described in Remark 3.2. It remains to show that the limiting element $(\tilde{\boldsymbol{\Omega}}, \tilde{\mathbf{f}})=:\left(\boldsymbol{\Omega}_{\beta, \gamma}^{\delta}, \mathbf{f}_{\beta, \gamma}^{\delta}\right)$ is a minimizer of the functional $J_{\beta, \gamma}$ with data $g^{\delta}$. This follows again analogously to the proof of Theorem 5.1, exploiting the lower-semicontinuity of norm and perimeter.

THEOREM 5.4 (Regularization property). Let $\left(\boldsymbol{\Omega}^{\dagger}, \mathbf{f}^{\dagger}\right) \in \mathcal{G}_{M}(D) \times \mathbb{R}^{m_{\dagger}}$ be given such that $f^{\dagger}=F\left(\boldsymbol{\Omega}^{\dagger}, \mathbf{f}^{\dagger}\right)$ fulfills $K f^{\dagger}=g$. For noisy data $g^{\delta} \in Y$ with $\left\|g^{\delta}-g\right\| \leq \delta$ choose the regularization parameters $\beta=\beta(\delta)$ and $\gamma=\gamma(\delta)$ according to

$$
\left.\begin{array}{l}
\beta(\delta) \rightarrow 0, \gamma(\delta) \rightarrow 0 \\
\frac{\delta^{2}}{\min (\beta(\delta), \gamma(\delta))} \rightarrow 0 \\
\frac{\max (\beta(\delta), \gamma(\delta))}{\min (\beta(\delta), \gamma(\delta))} \rightarrow 1
\end{array}\right\} \text { as } \delta \rightarrow 0
$$

For a sequence $\left(g^{\delta_{n}}, \delta_{n}\right)$ with $\left\|g^{\delta_{n}}-g\right\| \leq \delta_{n} \rightarrow 0$ let the pair $\left(\boldsymbol{\Omega}_{\beta_{n}, \gamma_{n}}^{\delta_{n}}, \mathbf{f}_{\beta_{n}, \gamma_{n}}^{\delta_{n}}\right)$ denote a minimizer of the Mumford-Shah functional $J_{\beta_{n}, \gamma_{n}}$ with data $g^{\delta_{n}}$ and the regularization parameter $\beta_{n}=\beta\left(\delta_{n}\right)$ and $\gamma_{n}=\gamma\left(\delta_{n}\right)$ chosen as above.

Then there exists a convergent subsequence of $\left(\boldsymbol{\Omega}_{\beta_{n}, \gamma_{n}}^{\delta_{n}}, \mathbf{f}_{\beta_{n}, \gamma_{n}}^{\delta_{n}}\right)$ with limit $(\tilde{\boldsymbol{\Omega}}, \tilde{\mathbf{f}})$. The associated function $\tilde{f}=F(\tilde{\boldsymbol{\Omega}}, \tilde{\mathbf{f}})$ is a solution of the equation $K \tilde{f}=g$. If the solution is unique then the whole sequence converges toward the solution.

Proof. Let a sequence $g^{\delta_{n}}$ be given with $\left\|g^{\delta_{n}}-g\right\| \leq \delta_{n} \rightarrow 0$ as $n \rightarrow \infty$ and let $\left(\beta_{n}, \gamma_{n}\right)$ be chosen according to the above parameter choice rule. We use the short 
notation $J_{n}:=J_{\beta_{n}, \gamma_{n}}, \boldsymbol{\Omega}^{n}:=\boldsymbol{\Omega}_{\beta_{n}, \gamma_{n}}^{\delta_{n}}$ and $\mathbf{f}^{n}:=\mathbf{f}_{\beta_{n}, \gamma_{n}}^{\delta_{n}}$. As $\left(\boldsymbol{\Omega}^{n}, \mathbf{f}^{n}\right)$ is a minimizer of the Mumford-Shah functional with data $g^{\delta_{n}}$ we have

$$
\begin{aligned}
\beta_{n}\left\|\mathbf{f}^{n}\right\|_{\ell_{2}}^{2}+\gamma_{n}\left|\partial \boldsymbol{\Omega}^{n}\right| & \leq\left\|K F\left(\boldsymbol{\Omega}^{n}, \mathbf{f}^{n}\right)-g^{\delta_{n}}\right\|^{2}+\beta_{n}\left\|\mathbf{f}^{n}\right\|_{\ell_{2}}^{2}+\gamma_{n}\left|\partial \boldsymbol{\Omega}^{n}\right| \\
& \leq\left\|K F\left(\boldsymbol{\Omega}^{\dagger}, \mathbf{f}^{\dagger}\right)-g^{\delta_{n}}\right\|^{2}+\beta_{n}\left\|\mathbf{f}^{\dagger}\right\|_{\ell_{2}}^{2}+\gamma_{n}\left|\partial \boldsymbol{\Omega}^{\dagger}\right| \\
& \leq \delta_{n}{ }^{2}+\beta_{n}\left\|\mathbf{f}^{\dagger}\right\|_{\ell_{2}}^{2}+\gamma_{n}\left|\partial \boldsymbol{\Omega}^{\dagger}\right| .
\end{aligned}
$$

In the same way we get

$$
\left\|K F\left(\boldsymbol{\Omega}^{n}, \mathbf{f}^{n}\right)-g^{\delta_{n}}\right\|^{2} \leq \delta_{n}{ }^{2}+\beta_{n}\left\|\mathbf{f}^{\dagger}\right\|_{\ell_{2}}^{2}+\gamma_{n}\left|\partial \boldsymbol{\Omega}^{\dagger}\right| .
$$

Using (5.5), it is

$$
\begin{aligned}
\min \left(\beta_{n}, \gamma_{n}\right)\left(\left\|\mathbf{f}^{n}\right\|_{\ell_{2}}^{2}+\left|\partial \boldsymbol{\Omega}^{n}\right|\right) & \leq \beta_{n}\left\|\mathbf{f}^{n}\right\|_{\ell_{2}}^{2}+\gamma_{n}\left|\partial \boldsymbol{\Omega}^{n}\right| \\
& \leq \delta_{n}{ }^{2}+\beta_{n}\left\|\mathbf{f}^{\dagger}\right\|_{\ell_{2}}^{2}+\gamma_{n}\left|\partial \boldsymbol{\Omega}^{\dagger}\right| \\
& \leq \delta_{n}{ }^{2}+\max \left(\beta_{n}, \gamma_{n}\right)\left(\left\|\mathbf{f}^{\dagger}\right\|_{\ell_{2}}^{2}+\left|\partial \boldsymbol{\Omega}^{\dagger}\right|\right) .
\end{aligned}
$$

It follows

$$
\left\|\mathbf{f}^{n}\right\|_{\ell_{2}}^{2}+\left|\partial \boldsymbol{\Omega}^{n}\right| \leq \frac{\delta_{n}{ }^{2}}{\min \left(\beta_{n}, \gamma_{n}\right)}+\frac{\max \left(\beta_{n}, \gamma_{n}\right)}{\min \left(\beta_{n}, \gamma_{n}\right)}\left(\left\|\mathbf{f}^{\dagger}\right\|_{\ell_{2}}^{2}+\left|\partial \boldsymbol{\Omega}^{\dagger}\right|\right) .
$$

With equation (5.7) and the parameter choice rule (5.4) the sequence of partitions $\left(\boldsymbol{\Omega}^{n}\right)_{n}$ has uniformly bounded perimeter and the sequence of coefficients is uniformly bounded,

$$
\left|\partial \boldsymbol{\Omega}^{n}\right| \leq C_{1} \quad \forall n \quad \text { and } \quad\left\|\mathbf{f}^{n}\right\| \leq C_{2} \quad \forall n .
$$

Again as in the previous proof we can extract a subsequence of $\left(\boldsymbol{\Omega}^{n}, \mathbf{f}^{n}\right)$, for simplicity also denoted by $\left(\boldsymbol{\Omega}^{n}, \mathbf{f}^{n}\right)$, such that

$$
\left(\boldsymbol{\Omega}^{n}, \mathbf{f}^{n}\right) \rightarrow(\tilde{\boldsymbol{\Omega}}, \tilde{\mathbf{f}}) \in \mathcal{G}_{M}(D) \times \mathbb{R}^{\tilde{m}} .
$$

Applying successively the lower-semicontinuity of norm and perimeter with respect to $d_{L_{1}}$, estimate (5.7) and the parameter choice rule (5.4) we get

$$
\begin{aligned}
\|\tilde{\mathbf{f}}\|_{\ell_{2}}^{2}+|\partial \tilde{\boldsymbol{\Omega}}| & \leq \lim \inf _{n \rightarrow \infty}\left(\left\|\mathbf{f}^{n}\right\|_{\ell_{2}}^{2}+\left|\partial \boldsymbol{\Omega}^{n}\right|\right) . \\
& \leq \lim \inf _{n \rightarrow \infty}\left(\frac{\delta_{n}{ }^{2}}{\min \left(\beta_{n}, \gamma_{n}\right)}+\frac{\max \left(\beta_{n}, \gamma_{n}\right)}{\min \left(\beta_{n}, \gamma_{n}\right)}\left(\left\|\mathbf{f}^{\dagger}\right\|_{\ell_{2}}^{2}+\left|\partial \boldsymbol{\Omega}^{\dagger}\right|\right)\right) \\
& =\left\|\mathbf{f}^{\dagger}\right\|_{\ell_{2}}^{2}+\left|\partial \boldsymbol{\Omega}^{\dagger}\right| .
\end{aligned}
$$

By (5.8) and Corollary 4.8 we have $f^{n}=F\left(\boldsymbol{\Omega}^{n}, \mathbf{f}^{n}\right) \stackrel{L_{2}}{\longrightarrow} F(\tilde{\boldsymbol{\Omega}}, \tilde{\mathbf{f}})=\tilde{f}$. As the operator $K$ is linear and bounded we get by the lower semicontinuity of the norm, estimate (5.6), the parameter choice rule (5.4) and the assumption $g^{\delta_{n}} \rightarrow g$

$$
\|K \tilde{f}-g\|_{L_{2}}^{2} \leq \lim \inf _{n \rightarrow \infty}\left\|K f^{n}-g^{\delta_{n}}\right\|_{L_{2}}^{2} \leq \lim \inf _{n \rightarrow \infty}\left(\delta_{n}{ }^{2}+\beta_{n}\left\|\mathbf{f}^{\dagger}\right\|_{\ell_{2}}^{2}+\gamma_{n}\left|\partial \boldsymbol{\Omega}^{n}\right|\right)=0 .
$$

Hence, it is $g=K \tilde{f}=K F(\tilde{\boldsymbol{\Omega}}, \tilde{\mathbf{f}})$ and $\tilde{f}$ is a solution to the equation. As $f^{\dagger}=F\left(\boldsymbol{\Omega}^{\dagger}, \mathbf{f}^{\dagger}\right)$ was an arbitrary solution and $\|\tilde{\mathbf{f}}\|_{\ell_{2}}^{2}+|\partial \tilde{\boldsymbol{\Omega}}| \leq\left\|\mathbf{f}^{\dagger}\right\|_{\ell_{2}}^{2}+\left|\partial \boldsymbol{\Omega}^{\dagger}\right|$ we have found a solution with minimal penalty. 
6. SPECT - an application from medical imaging. In this section we present a numerical verification of our main result, Theorem 5.4, which states that we reconstruct the right segmentation and the exact coefficients only if the noise in the data is small enough and the regularization parameters are chosen according to the given parameter choice rule (5.4). The conditions of equation (5.4) are readily fulfilled if we choose

$$
\beta(\delta)=c_{1} \gamma(\delta)=c_{2} \delta^{\nu}
$$

with constants $c_{1}, c_{2}>0$ and an exponent $1 \leq \nu<2$. For our test computations we have chosen $c_{1}=1, c_{2}=0.01$ and $\nu=1.9$.

The results of the previous sections are formulated for arbitrary linear operators. Here, we will consider the reconstruction and segmentation of an activity distribution $f$ from SPECT data with known density function $\mu$. SPECT stands for Single Photon Emission Computerized Tomography [8, 39, 41, 18] and is an imaging method designed to provide information about the functional level of a part of the body. SPECT involves the injection of a low-level radioactive chemical, called radiotracer or radiopharmaceutical into the bloodstream. The radiotracer travels in the bloodstream and accumulates, e.g., in the heart or it can be attached to certain types of proteins which are known to bind to tumor cells. The concentration of the radiopharmaceutical within the body is referred to as activity distribution $f$. The radioactive material ejects photons which travel through the body and interact with the tissue, where the interaction (i.e., the absorption of photons) depends on the mass density of the tissue $\mu$. Finally the photons are measured outside the body by a SPECT scanner (a $\gamma$-camera). The resulting sinogram data $y$ is modeled by the attenuated Radon transform,

$$
g(s, \omega)=A_{\mu} f(s, \omega)=\int_{\mathbb{R}} f\left(s \omega+t \omega^{\perp}\right) \exp \left(\int_{\tau=-t}^{\infty}-\mu\left(s \omega+\tau \omega^{\perp}\right)\right) d t
$$

with $(s, \omega) \in \mathbb{R} \times S^{1}$ where $S^{1}$ is the one-dimensional unit sphere. The attenuated Radon transform is linear with respect to the activity distribution $f$ and in this case, exact inversion formulae exist $[25,26,39]$. However, due to the measurement process, the given data will be noisy and hence, also for the linear attenuated Radon transform considered as operator $A_{\mu}: L_{2}\left(\mathbb{R}^{2}\right) \rightarrow L_{2}\left(\mathbb{R} \times S^{1}\right)$ regularization methods have to be used [14, 21]. Due to the underlying physical process (photons are emitted, interact, travel and are finally counted) the noise in SPECT data follows a Poisson distribution $[37,15]$.

In order to support Theorem 5.4 we need data with a noise level that tends to zero. In general, the only way to reduce the effect of Poisson noise (also called photon noise or shot noise) is to capture more signal. In practice, this can be realized by a longer exposure time, but still, the number of photons captured is limited by the capacity of the sensor. As mentioned above, the noise in the SPECT measurements follows a Poisson distribution. For our numerical tests, however, the data was contaminated with multiplicative white noise, which resembles important properties of Poisson noise, e.g. that the amount of noise is related to the intensity of the signal, see Figure 6.2.

As test functions we choose activity and density distributions as shown in Figure 6.1. The density distribution is an ellipsoid with constant value 1 and assumed to be known. The activity distribution consists of five different objects: a square with value 8 , two different sized diamonds with values 3 and 4 for the bigger and the 

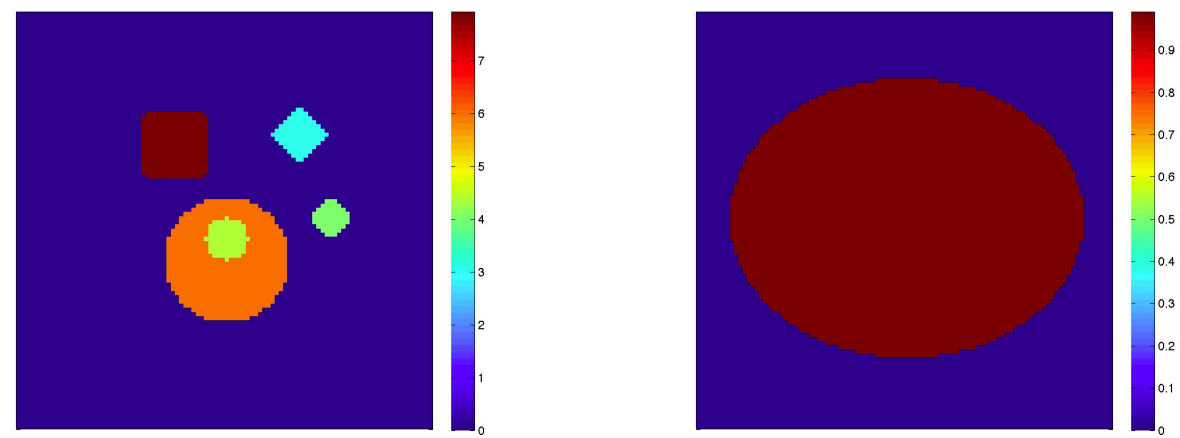

FIG. 6.1. Left: The (unknown) activity function $f$ with five domains: square with value 8, big diamond with value 3, small diamond with value 4, big circle with value 6 and circular inclusion with value 5. Middle: The (known) density function $\mu$ : ellipsoid with value 1 .
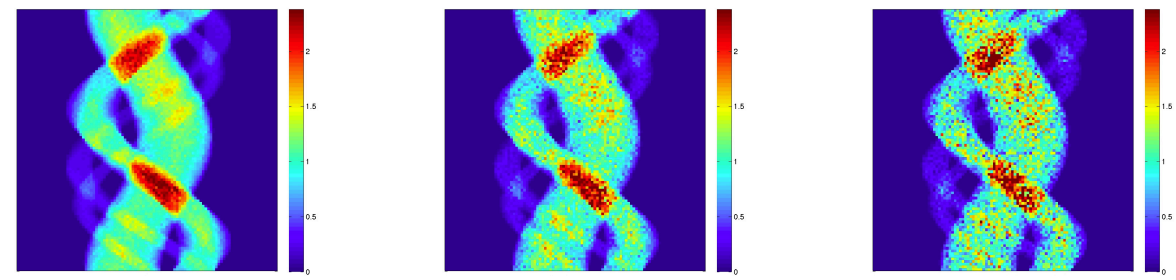

FIG. 6.2. SPECT data with different amounts of multiplicative noise. From left to right: 5, 10 and $15 \%$.

smaller one respectively, a big circle with value 6 with an off-center circular inclusion with value 5 .

With a Matlab-implementation of the attenuated Radon transform (6.1) sinogram data are generated and perturbed by multiplicative noise with different error level. The approximate solution $\left(\boldsymbol{\Omega}_{\beta, \gamma}^{\delta}, \mathbf{f}_{\beta, \gamma}^{\delta}\right)$ is calculated as minimizer of the Mumford-Shah type functional $J_{\beta, \gamma}$ with the algorithm as described in [31]. We briefly repeat the most important steps of the algorithm. The shape variable $\boldsymbol{\Omega}$ is encoded as the zero level-set of a level-set function $\phi[28,36]$. The update of the coefficients and the shape variable is done in an alternating fashion: first, the shape variable $\boldsymbol{\Omega}$ is fixed and optimal coefficients are computed as described in Proposition 4.1. Second, the coefficients are fixed and the shape is updated using shape optimization techniques [6, 17, 31, 40]. For that, we compute the shape derivative $d_{\Gamma}$ of the reduced functional (i.e., we consider only the geometry $\boldsymbol{\Omega}$ as free variable)

$$
\hat{J}(\boldsymbol{\Omega})=\left\|A_{\mu}(f)-g^{\delta}\right\|_{L_{2}\left(\mathbb{R} \times S^{1}\right)}^{2}+\beta\|\mathbf{f}(\boldsymbol{\Omega})\|_{\ell_{2}}^{2}+\gamma|\partial \boldsymbol{\Omega}|
$$


and get

$$
\begin{aligned}
d_{\Gamma}(\hat{J}, F)=2 \sum_{i=1}^{n} \mathrm{f}_{i} \int_{x \in \partial \Omega_{i}} A_{\mu}^{*}\left(A_{\mu}(f)\right. & \left.-g^{\delta}\right)(x) F(x) d S(x) \\
& +\beta \sum_{i=1}^{n} \mathrm{f}_{i}^{2} \int_{x \in \partial \Omega_{i}} F(x) d S(x)+\gamma \int_{\partial \boldsymbol{\Omega}} \kappa d S .
\end{aligned}
$$

Choosing a descent direction $F$, i.e., an $F$ such that $d_{\Gamma}(\hat{J}, F)<0$, enables us to update the geometry by propagating the associated level-set function $\phi$ by solving the level-set equation

$$
\frac{\partial \phi}{\partial t}+F|\nabla \phi|=0
$$

As the level-set evolution might get stuck in local minima we also use an insert strategy to create new components based on the functional derivative of the data fit term. For a detailed description of the algorithm we refer to [31, 19].

The unknown (as well as the known) distribution (Figure 6.1) has dimension $101 \times 101$ pixels. Its sinogram was computed for 101 samples and 100 angles. We have calculated reconstructions from data with relative error levels

$$
\delta_{\text {rel }} \in\{0 \%, 0.005 \%, 0.01 \%, \ldots, 30 \%\} .
$$

The distance of the associated reconstruction to the original function is measured in the $L_{1}$-norm. Figure 6.3 shows the values of the Mumford-Shah type functional evaluated at the computed minimizers $f_{\beta, \gamma}^{\delta}$ (left image) and the relative $L_{1}$-accuracy $\left\|f-f_{\beta, \gamma}^{\delta}\right\|_{L_{1}} /\|f\|_{L_{1}}$ of the computed minimizers $f_{\beta, \gamma}^{\delta}$ of the Mumford-Shah type functional (right image). With decreasing noise level also the values of the functional and the reconstruction error decrease. The Mumford-Shah type functional evaluated at the computed minimizers decrease monotonically (apart from two outliers) with the error level (Figure 6.3, left) whereas the relative $L_{1}$-accuracy of the computed minimizers only shows in average a decreasing behavior (Figure 6.3, right). The frequently observed outliers are due to the fact that single pixels, that are assigned to the wrong set, have a greater influence in the $L_{1}$-norm than in the Mumford-Shah type functional.

Figure 6.4 and Figure 6.5 show reconstructions and segmentations from data with $1 \%, 5 \%, 10 \%, 15 \%, 20 \%$ and $25 \%$ noise. The iteration was stopped when one of the following criteria was fulfilled

- The residual error of the current approximation $f^{n}$ is of the size of the data error, i.e., the iteration was carried out as long as $\left\|A_{\mu}\left(f^{n}\right)-g^{\delta}\right\|>\tau\left\|g-g^{\delta}\right\|$ with $\tau=1.1$.

- The maximal number of outer iterations (100) was reached. (With outer iteration we refer to one complete update of both the coefficients and the geometry. For the geometry update another inner iteration, namely time stepping for the solution of the level set equation, is done for each outer iteration.)

For the lower error levels up to $6 \%$ relative noise, the maximal number of iterations was used. For the larger error levels, the number of iterations varied between 40 and 75 , decreasing with increasing error level.

Figures 6.4 and 6.5 clearly demonstrate that the reconstructions from noisy data converge as the noise level gets smaller, which is in accordance to Theorem 5.4. For 

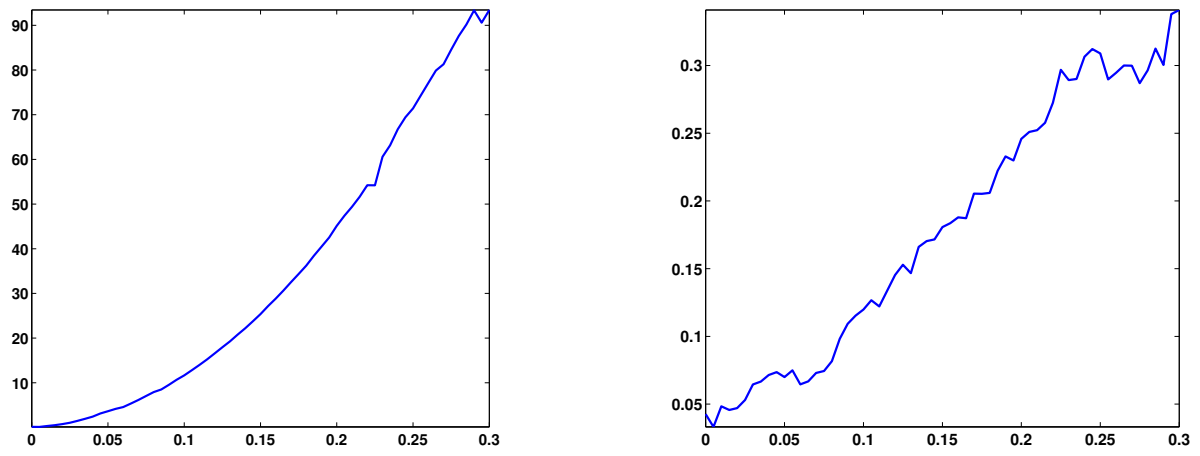

FiG. 6.3. Left: Values of the Mumford-Shah type functional evaluated at the computed minimizers $f_{\beta, \gamma}^{\delta}$. Right: Relative reconstruction error $\left\|f-f_{\beta, \gamma}^{\delta}\right\|_{L_{1}} /\|f\|_{L_{1}} ;$ both for data with relative error level between 0 and $30 \%$.

high noise levels, the reconstruction misses a whole component and the values of $f$ are slightly off. Furthermore, one can see some oscillations in the segmenting contour (e.g., the square in Figure 6.5, upper left). However, the smaller the noise in the data, the better the reconstruction for both the shapes and the values, until we get an almost perfect reconstruction from data with $1 \%$ noise. The oscillations in the segmenting contour could be prevented by putting a higher weight on the perimeter penalty. However, there is always a trade-off between small details such as the circular inclusion and smooth boundaries. The circular inclusion, which has a very low contrast difference to the background, is detected for noise up to $8 \%$, for higher noise level the inclusion is not detected, at least not for the chosen set of parameters. This is mainly due to the influence of the perimeter penalty: for large regularization parameters (i.e., high noise), the influence of the perimeter constraint increases, leading to vanishing small sets, in particular if the contrast difference to the background is small.

As a second, 'real world', example we have performed test computations for activity and density functions specified by the standard mathematical cardiac torso (MCAT) phantom [38, 41] as shown in Figure 6.6. The piecewise constant activity function $f$ represents a section through a simplified model of a human heart. Figure 6.6(left and right) shows the blood supply of the myocardal muscle (no.2) and the two ventricles (no.1 and no.3). The blood supply is interrupted at one point, namely in the outer left area of the myocardal muscle. This point is not reached by the radiopharmaceuticum, hence the left ventricle seems to be 'open'. For that reason, the outer area (no.1) and the upper cardic ventricle have the same number and are modeled as one connected domain in the $P C_{m}$-model.

Even with a high noise level in the data we obtain an excellent reconstruction and segmentation, see Figure 6.7. However, it is more difficult to see the regularization property of our approach. This might be due to the fact that the activity function of Figure 6.6 only consists of very few pixels. This means that the discretization error dominates in the reconstruction.

\section{REFERENCES}

[1] L. Ambrosio, N. Fusco, and D. Pallara. Functions of Bounded Variation and Free Discontinuity 

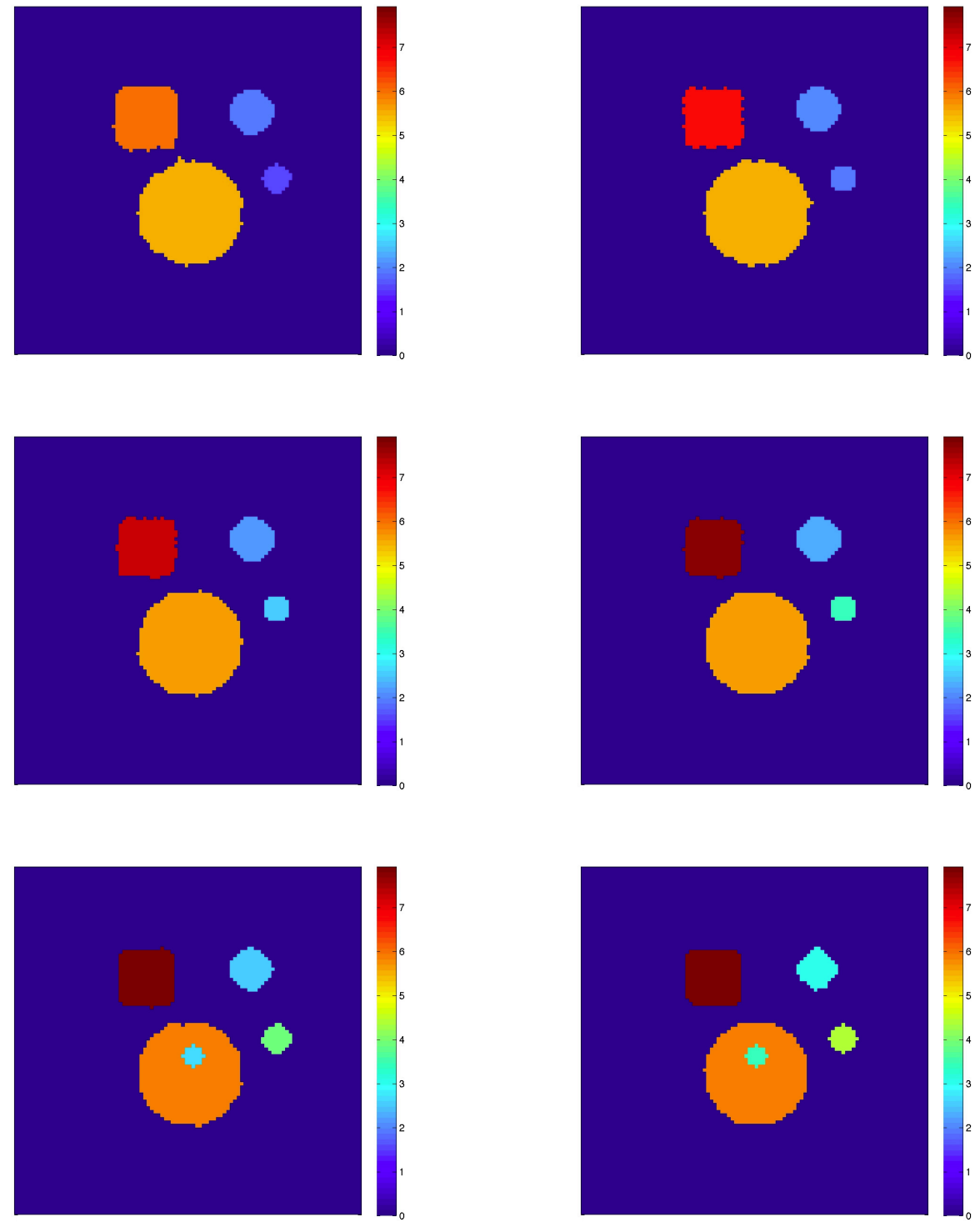

FIG. 6.4. Reconstructions from data with relative error level 25\% (upper left), $20 \%$ (upper right), $15 \%$ (middle left), $10 \%$ (middle right), $5 \%$ (lower left) and 1\% (lower right).

Problems. Oxford University Press, Oxford, 2000.

[2] L. Bar, N. Sochen, and N. Kiryati. Semi-blind image restoration via Mumford-Shah regularization. Image Processing, IEEE Transactions on, 15(2):483-493, 2006.

[3] Hend Ben Ameur, Martin Burger, and Benjamin Hackl. Level set methods for geometric inverse problems in linear elasticity. Inverse Problems, 20(3):673-696, 2004.

[4] M. Burger. A level set method for inverse problems. Inverse Problems, 17:1327-1355, 2001.

[5] Antonin Chambolle. Image segmentation by variational methods: Mumford and Shah functional and the discrete approximations. SIAM J. Appl. Math., 55(3):827-863, 1995.

[6] T. F. Chan and L. A. Vese. A level set algorithm for minimizing the Mumford-Shah functional in image processing. UCLA CAM Report 00-13, University of California, Los Angeles, 

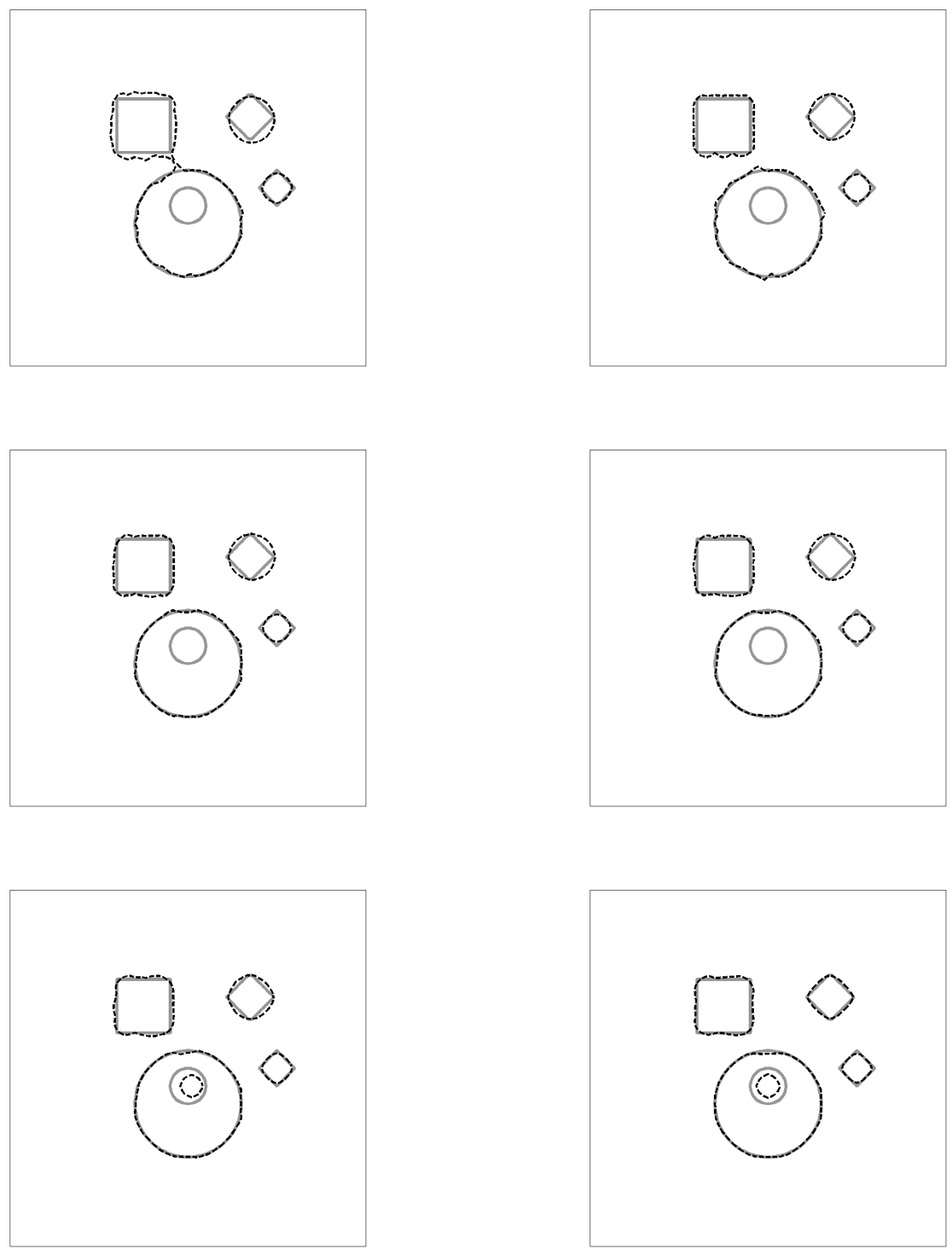

FIG. 6.5. Segmentations (true: solid, grey, reconstructed: black, dashed) from data with relative error level 25\% (upper left), 20\% (upper right), 15\% (middle left), 10\% (middle right), 5\% (lower left) and $1 \%$ (lower right).

2000.

[7] T. F. Chan and L. A. Vese. Active contours without edges. IEEE Trans. Image Processing, 10(2):266-277, 2001.

[8] S.R. Cherry, J.A. Sorenson, and M.E. Phelps. Physics in Nuclear Medicine. Saunders, 2003.

[9] Guy David. Singular sets of minimizers for the Mumford-Shah functional. Progress in Mathematics 233. Basel: Birkhäuser. xiv, 581 p. EUR 115.56 , 2005.

[10] A DeCezaro, A Leit ao, and X-C Tai. On multiple level-set regularization methods for inverse problems. Inverse Problems, 25(3), 2009.

[11] M. C. Delfour and J.-P. Zolésio. Shapes and geometries. Society for Industrial and Applied 

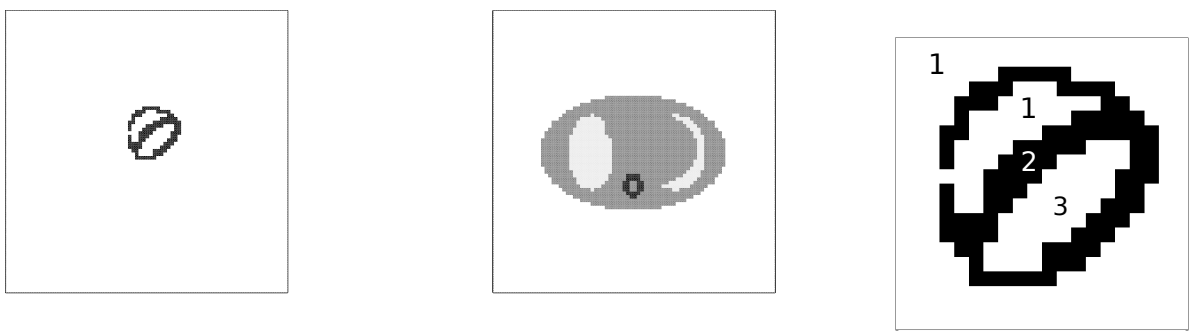

FIG. 6.6. Activity function $f$ and density function $\mu$ specified by the MCAT phantom. Left: activity function as a section through the heart. Middle: density function as a section through a human torso. Right: zoom into activity function and labeling of the domains.
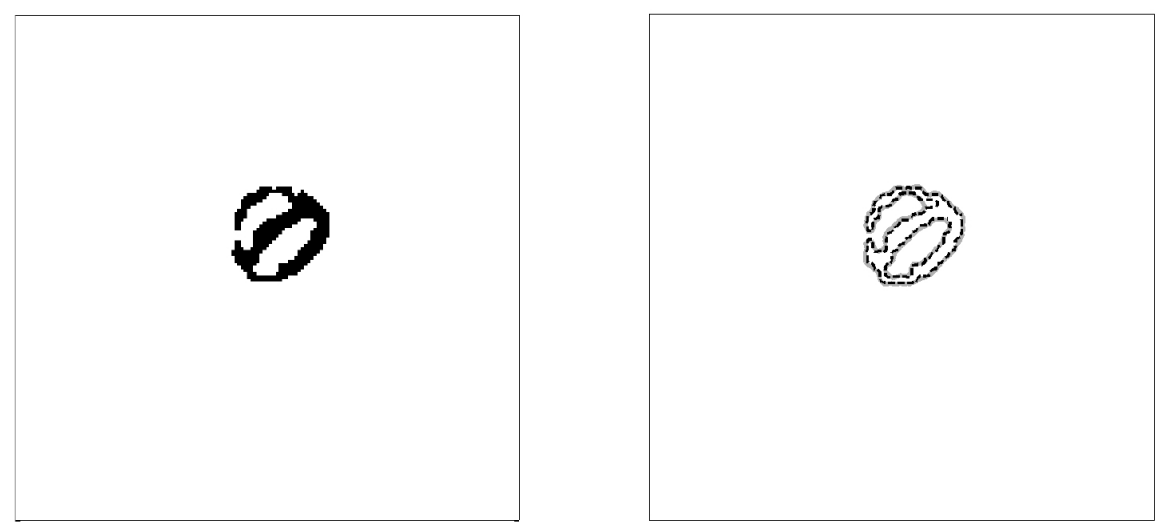

FIG. 6.7. Results of the Mumford-Shah type method from data with $15 \%$ relative noise: Left: reconstruction. Right: segmentation; original contours: gray, solid; reconstructed contours: black, dashed.

Mathematics (SIAM), Philadelphia, PA, 2001. Analysis, differential calculus, and optimization.

[12] Marc Droske and Wolfgang Ring. A Mumford-Shah level-set approach for geometric image registration. SIAM J. Appl. Math., 66(6):2127-2148 (electronic), 2006.

[13] A. Frühauf, F.Leitao and O. Scherzer. Analysis of regularization methods for the solution of ill-posed problems involving discontinuous operators. SIAM J. Num. Anal., 43:767-786, 2005.

[14] J.-P. Guillement, F. Jauberteau, L. Kunyansky, R. Novikov, and R. Trebossen. On singlephoton emission computed tomography imaging based on an exact formula for the nonuniform attenuation correction. Inverse Probl., 18(6), 2002.

[15] J-P Guillement and R G Novikov. A noise property analysis of single-photon emission computed tomography data. Inverse Problems, 20(1):175, 2004.

[16] M. Hintermüller and K. Kunisch. Totally bounded variation regularization as a bilaterally constrained optimization problem. SIAM Journal on Applied Mathematics, 64(4):1311$1333,2004$.

[17] M. Hintermüller and W. Ring. An inexact Newton-CG-type active contour approach for the minimization of the Mumford-Shah functional. J. Math. Imag. Vis., 20(1-2):19-42, 2004.

[18] A.C. Kak and Malcom Slaney. Principles of Computerized Tomographic Imaging. Society of Industrial and Applied Mathematics, 2001.

[19] Esther Klann, Ronny Ramlau, and Wolfgang Ring. A Mumford-Shah level-set approach for the inversion and segmentation of X-ray tomography data. Inverse Problems and Imaging, 30(1):137-166, 2011.

[20] R. Kress. Linear Integral Equations. Springer, Berlin, 1999.

[21] Leonid A. Kunyansky. A new SPECT reconstruction algorithm based on the Novikov explicit 
inversion formula. Inverse Probl., 17(2):293-306, 2001.

[22] A. Leitao and O. Scherzer. On the relation between constraint regularization, level sets, and shape optimization. Inverse Problems, 19:L1-L11, 2003.

[23] A. Litman, D. Lesselier, and F. Santosa. Reconstruction of a two-dimensional binary obstacle by controlled evolution of a level-set. Inverse Problems, 14:685-706, 1998.

[24] David Mumford and Jayant Shah. Optimal approximations by piecewise smooth functions and associated variational problems. Comm. Pure Appl. Math., 42(5):577-685, 1989.

[25] F. Natterer. Inversion of the attenuated Radon transform. Inverse Probl., 17(1):113-119, 2001.

[26] Roman G. Novikov. An inversion formula for the attenuated $X$-ray transformation. Ark. Mat., 40(1):145-167, 2002.

[27] Stanley Osher and James A. Sethian. Fronts propagating with curvature-dependent speed: algorithms based on Hamilton-Jacobi formulations. J. Comput. Phys., 79(1):12-49, 1988.

[28] Stanley J. Osher and Ronald P. Fedkiw. Level Set Methods and Dynamic Implicit Surfaces. Springer Verlag, New York, 2002.

[29] Stanley J. Osher and Fadil Santosa. Level set methods for optimization problems involving geometry and constraints. I. Frequencies of a two-density inhomogeneous drum. J. Comput. Phys., 171(1):272-288, 2001.

[30] T. Pock, A. Chambolle, H. Bischof, and D. Cremers. A convex relaxation approach for computing minimal partitions. In IEEE Conference on Computer Vision and Pattern Recognition (CVPR), Miami, Florida, 2009.

[31] Ronny Ramlau and Wolfgang Ring. A Mumford-Shah level-set approach for the inversion and segmentation of X-ray tomography data. J. Comput. Phys., 221(2):539-557, 2007.

[32] Ronny Ramlau and Wolfgang Ring. Regularization of linear operator equations by perimeter constraints. Inverse Problems, 26(11), 2010.

[33] Luca Rondi and Fadil Santosa. Enhanced electrical impedance tomography via the MumfordShah functional. ESAIM Control Optim. Calc. Var., 6:517-538 (electronic), 2001.

[34] L.T Rudin, S. Osher, and E. Fatemi. Nonlinear total variation based noise removal algorithm. Physica D, 60:259-268, 1992.

[35] F. Santosa. A level-set approach for inverse problems involving obstacles. ESAIM: Control, Optimization and Calculus of Variations, 1:17-33, January 1996.

[36] J. A. Sethian. Level set methods and fast marching methods. Cambridge University Press, Cambridge, second edition, 1999. Evolving interfaces in computational geometry, fluid mechanics, computer vision, and materials science.

[37] L. A. Shepp and Y. Vardi. Maximum Likelihood Reconstruction for Emission Tomography. IEEE Transactions on Medical Imaging, 1(2):113-122, 1982.

[38] J.A. Terry, B.M.W. Tsui, J.R. Perry, J.L. Hendricks, and G.T. Gullberg. The design of a mathematical phantom of the upper human torso for use in 3-d spect imaging research. In: Proc. 1990 Fall Meeting Biomed. Eng. Soc., Blacksburg, VA, New York University Press., pages 1467-1474, 1990.

[39] Oleh Tretiak and Charles Metz. The exponential Radon transform. SIAM J. Appl. Math., 39:341-354, 1980.

[40] A. Tsai, A. Yezzi, and A. S. Willsky. Curve evolution implementation of the Mumford-Shah functional for image segmentation, denoising, interpolation, and magnification. IEEE Transactions on image processing, 10(8):1169-1186, 2001.

[41] M.N. Wernick and J.N. (eds.) Aarsvold. Emission Tomography. The Fundamentals of PET and SPECT. Elsevier Academic Press, 2004. 